\title{
A multifrequency angular power spectrum analysis of the Leiden polarization surveys
}

\author{
L. La Porta ${ }^{1, \star}$ and C. Burigana ${ }^{2}$ \\ 1 Max Planck Institut für Radioastronomie, Auf dem Hügel 69, 53121 Bonn, Germany \\ e-mail: laporta@mpifr-bonn.mpg.de \\ 2 INAF - IASF Bologna, via P. Gobetti 101, 40129 Bologna, Italy \\ e-mail: burigana@iasfbo.inaf.it
}

Received 7 October 2005 / Accepted 15 May 2006

ABSTRACT

\begin{abstract}
The Galactic synchrotron emission is expected to be the most relevant source of astrophysical contamination in cosmic microwave background polarization measurements, at least at frequencies $v \lesssim 70 \mathrm{GHz}$ and at angular scales $\theta \gtrsim 30^{\prime}$. We present a multifrequency analysis of the Leiden surveys, linear polarization surveys covering the Northern Celestial Hemisphere at five frequencies between $408 \mathrm{MHz}$ and $1411 \mathrm{MHz}$. By implementing specific interpolation methods to deal with these irregularly sampled data, we produced maps of the polarized diffuse Galactic radio emission with a pixel size $\simeq 0.92^{\circ}$. We derived the angular power spectrum (APS) ( $P I, E$, and $B$ modes) of the synchrotron dominated radio emission as function of the multipole, $\ell$. We considered the whole covered region and some patches at different Galactic latitudes. By fitting the APS in terms of power laws $\left(C_{\ell} \sim \kappa \cdot \ell^{\alpha}\right)$, we found spectral indices that steepen with increasing frequency: from $\alpha \sim-(1-1.5)$ at $408 \mathrm{MHz}$ to $\alpha \sim-(2-3)$ at $1411 \mathrm{MHz}$ for $10 \lesssim \ell \lesssim 100$ and from $\alpha \sim-0.7$ to $\alpha \sim-1.5$ for lower multipoles (the exact values depending on the considered sky region and polarization mode). The bulk of this flattening at lower frequencies can be interpreted in terms of Faraday depolarization effects. We then considered the APS at various fixed multipoles and its frequency dependence. Using the APSs of the Leiden surveys at $820 \mathrm{MHz}$ and $1411 \mathrm{MHz}$, we determined possible ranges for the rotation measure, $R M$, in the simple case of an interstellar medium slab model. Also taking into account the polarization degree at $1.4 \mathrm{GHz}$, it is possible to break the degeneracy between the identified $R M$ intervals. The most reasonable of them turned out to be $R M \sim 9-17 \mathrm{rad} / \mathrm{m}^{2}$ although, given the uncertainty on the measured polarization degree, $R M$ values in the interval $\sim 53-59 \mathrm{rad} / \mathrm{m}^{2}$ cannot be excluded.
\end{abstract}

Key words. polarization - galaxy: general - cosmology: cosmic microwave background - methods: data analysis

\section{Introduction}

In the last decade an impressive number of experiments has been dedicated to measurements of the cosmic microwave background $(\mathrm{CMB})$ anisotropies, first observed in temperature by COBE (Smoot et al. 1990). Modern cosmologies predict also the existence of polarization fluctuations, produced at the recombination epoch via Thomson scattering (see, e.g., Kosowski 1999). However, the foreseen degree of polarization of the CMB anisotropies should be $\$ 10 \%$, implying a signal much weaker than in temperature and therefore extremely difficult to reveal. The first detection of the $\mathrm{CMB}$ polarization achieved by DASI (Kovac 2002) and the recent measure by BOOMERanG (Masi et al. 2006; Montroy et al. 2005) confirmed the expectations. The polarization data expected by the NASA WMAP ${ }^{1}$ satellite will permit us to extend the information about polarization to a wider portion of the sky, though at small scales $\left(\theta \lesssim 0.5^{\circ}\right)$ its sensitivity will likely allow a detection rather than an accurate measure.

In the near future, the ESA PLANCK ${ }^{2}$ satellite will significantly improve these results by observing the whole sky at nine

\footnotetext{
* Member of the International Max Planck Research Shool (IMPRS) for Radio and Infrared Astronomy at the Universities of Bonn and Cologne.

${ }^{1}$ http://lambda.gsfc.nasa.gov/product/map/

${ }^{2}$ http://www.rssd.esa.int/planck
}

different frequencies between 30 and $857 \mathrm{GHz}$, both in temperature and polarization, with unprecedented resolution and sensitivity (e.g. see Tauber 2004, and references therein). In particular, the sensitivities per pixel of the PLANCK maps are expected to be between 3-4 and 10 times better than in the WMAP data, producing a significant improvement in terms of angular power spectrum recovery. Consequently, PLANCK alone will allow an estimate of the cosmological parameters much more precise than that obtained by WMAP and other CMB experiments combined with different cosmological data. Moreover, it will accurately measure the CMB $E$-mode and could potentially observe the $B$-mode as well (e.g. see Burigana et al. 2004, and references therein).

Strong efforts have been made to evaluate the impact of the foregrounds on the feasibility of CMB anisotropy measurements, as well as to understand how to separate the different components contributing to the observed maps. The methods elaborated for this purpose can be divided in two categories: blind (Independent Component Analysis, see Baccigalupi et al. 2000; Maino et al. 2002; Baccigalupi et al. 2004; Delabrouille et al. 2003) and non-blind (Wiener filtering and Maximum Entropy Methods, see respectively Bouchet et al. 1999 and Hobson et al. 1998). The latter class needs an a priori knowledge of the frequency and spatial dependence of the foreground properties, while a blind approach only requires maps at different frequencies as input. On the other hand, blind methods 
benefit from having ancillary data (such as realistic templates) to improve the separation robustness and quality ${ }^{3}$.

The Galactic polarized diffuse synchrotron radiation is expected to play a major role at frequencies below $70 \mathrm{GHz}$ on intermediate and large angular scales $\left(\theta \gtrsim 30^{\prime}\right)$, at least at medium and high Galactic latitudes where satellites have the clearest view of the $\mathrm{CMB}$ anisotropies. At about $1 \mathrm{GHz}$ the synchrotron emission is the most important radiative mechanism out of the Galactic plane, while at low latitudes it is comparable with the bremsstrahlung; however the free-free emission is unpolarized, whereas the synchrotron radiation could reach a theoretical intrinsic degree of polarization of about $75 \%$. Consequently, radio frequencies are the natural range for studying the Galactic synchrotron emission, although it might be affected by Faraday rotation and depolarization. Nowadays ${ }^{4}$ the only available data suitable for studying the polarized Galactic synchrotron emission on large scales are the so-called Leiden surveys (Brouw \& Spoelstra 1976). These are linear polarization surveys, extending up to high Galactic latitudes, carried out at five frequencies between $408 \mathrm{MHz}$ and $1411 \mathrm{MHz}$. We have elaborated specific interpolation methods to project these surveys into maps with a pixel size $\simeq 0.92^{\circ}$. These maps can be exploited in different contexts. They are suitable to analyse the statistical properties of the Galactic radio polarized emission. Beside this obvious application, they can be used as input to construct templates for simulation activities in the context of current and future microwave polarization anisotropy experiments. For example they can be utilized to build templates for straylight evaluation (see, e.g., Challinor et al. 2000; Burigana et al. 2001; Barnes et al. 2003) and for component separation analyses.

It is standard practice to characterize the CMB anisotropies in terms of the angular power spectrum ${ }^{5}$ (APS) as a function of the multipole, $\ell$ (inversely proportional to the angular scale, $\left.\ell \simeq 180 / \theta\left(^{\circ}\right)\right)$. The APS is an estimator related to the angular two-point correlation function of the fluctuations of a sky field (Peebles 1993). The APS is not able to fully characterize the complexity of the intrinsically non-Gaussian Galactic emission. Nevertheless, it is of increasing use also in the study of the correlation properties of the Galactic foregrounds (see, e.g., Baccigalupi et al. 2001, for an application to the polarized radio emission). We stick to the commonly adopted approach and consider the APS of both the polarized intensity, $P I$, and the $E$ and $B$ modes $^{6}$ (see Kamionkowski et al. 1997; and Zaldarriaga 2001).

\footnotetext{
${ }^{3}$ Future component separation methods will probably search for a good compromise between the relaxation of the a-priori assumptions on the independent signals superimposed on the microwave sky and the exploitation of the physical correlation among the various components. Also it will be crucial to analyse microwave data together with ancillary data from radio and infrared frequencies.

${ }^{4}$ During the final phase of the revision process of this work, the DRAO $1.4 \mathrm{GHz}$ polarization survey has been released (Wolleben et al. 2006). The data are available at http://www.mpifr-bonn.mpg.de/div/konti/26msurvey/ or http://www.drao-ofr.hia-iha.nrc-cnrc.gc.ca/26msurvey/ or http://cdsweb.u-strasbg.fr/cgi-bin/qcat/?J/A+A/448/411. See La Porta et al. (2006) for a first angular power spectrum analysis of the DRAO survey.

${ }^{5}$ It contains all the relevant statistical information in the case of pure Gaussian fluctuations.

${ }^{6}$ We keep here the physical dimension of the maps (antenna temperature, typically in $\mathrm{K}$ or $\mathrm{mK}$ ) and consequently express the APS in $\mathrm{K}^{2}$ or $\mathrm{mK}^{2}$.
}

In Sect. 2 we briefly summarize the properties of the Leiden surveys and of some selected areas relevant for the analysis at the smaller angular scales. In Sect. 3 we introduce the general criteria adopted to elaborate the class of our interpolation algorithms. The final, optimized version of the algorithm and the accuracy of the derived maps and APSs are described in Sect. 4. In Sect. 5 we present our results in terms of maps, APSs, and a parametric description of the APSs. A brief comparison with a preliminary version of the DRAO polarization survey (Wolleben et al. 2003; Wolleben 2005) at $1.4 \mathrm{GHz}$ is given in Sect. 6 in terms of APS. Section 7 is devoted to the multifrequency analysis of our results and to their interpretation in the context of an interstellar medium slab model. We summarize and discuss our main results in Sect. 8.

\section{The Leiden surveys and selected areas}

The so-called Leiden surveys are the final results of different observational campaigns carried out in the sixties with the 25-m radio telescope at Dwingeloo. The complete data sets as well as the observation reduction and calibration methods have been presented by Brouw \& Spoelstra (1976). The observations were performed at $408,465,610,820$ and $1411 \mathrm{MHz}$ with an angular resolution of $\theta_{\mathrm{HPBW}}=2.3^{\circ}, 2.0^{\circ}, 1.5^{\circ}, 1.0^{\circ}, 0.6^{\circ}$, respectively. At each frequency, the observed positions are inhomogenously spread over the Northern Galactic Hemisphere and globally cover a sky fraction of about $\sim 50 \%$. The errors, quoted for points observed at least twice, follow Gaussian distributions with mean values of $0.34,0.33,0.16,0.11$ and $0.06 \mathrm{~K}$ from the lower to the higher frequency, respectively. The observations at the same position were taken at different azimuths, elevations, and days (the adopted scanning strategy being random in coordinates and time). A robust control and removal of the ground radiation contaminating the observations have been also performed. These properties imply a very low level of contamination from residual systematic effects, at least in comparison with the intrinsic survey sensitivities, and make these surveys a reference point (though insufficient by itself) for absolute calibration of radio data. As an example, the absolute calibration of the Effelsberg maps at $1.4 \mathrm{GHz}$ has been performed using the Leiden survey at $1411 \mathrm{MHz}$ (see Uyaniker et al. 1998, for a description of the procedure).

The main problem in the analysis of the Leiden surveys is their poor sampling across the sky; it is necessary to project them onto maps with pixel size of about $3.7^{\circ}$ (we use here the HEALPix scheme, see Górski et al. 2005) to find at least one observation on each pixel of the observed region, that would limit the angular power spectrum recovery to $\ell \simeq 50$. On the other hand, for some sky areas the sampling is significantly better than the average, by a factor $\simeq 4$, and the maps appear filled even for a pixel size of $\sim 1.8^{\circ}$, allowing one to reach multipoles $\ell \simeq 100$. We identified three regions that permit one to investigate this multipole range at both low and middle/high Galactic latitudes: patch $1\left[\left(110^{\circ} \leq l \leq 160^{\circ}, 0^{\circ} \leq b \leq 20^{\circ}\right)\right]$; patch 2 $\left[\left(5^{\circ} \leq l \leq 80^{\circ}, b \geq 50^{\circ}\right)\right.$ together with $\left(0^{\circ} \leq l \leq 5^{\circ}, b \geq 60^{\circ}\right)$ and $\left.\left(335^{\circ} \leq l \leq 360^{\circ}, b \geq 60^{\circ}\right)\right]$; patch $3\left[\left(10^{\circ} \leq l \leq 80^{\circ}\right.\right.$, $\left.\left.b \geq 70^{\circ}\right)\right]$.

We underline that in these patches the polarized signal and the corresponding signal-to-noise ratio are typically higher than the average; they are associated with the brightest structures of the polarized radio sky, i.e. the "Fan Region" (patch 1) and the "North Polar Spur" (patch 2 and 3). The "North Polar Spur" (NPS) has been extensively studied (see Salter 1983; and Egger \& Aschenbach 1995). The theories that better match the 
observations are all variants of the same model and interpret the NPS as the front shock of an evolved Supernova Remnant (SNR), whose distance should be $\sim 100 \pm 20$ pc (inferred from starlight polarization, see Bingham 1967). In contrast, the present knowledge of the "Fan Region" is much poorer. A rough estimate of its distance can be derived from purely geometrical considerations: it has such an extent on the sky that it must be located within $(1-2) \times 10^{2} \mathrm{pc}$ from the Sun not to have an unrealistically large dimension. Wolleben et al. (2005) has recently proposed a different view of the "Fan Region": from the distances of HII regions that they consider to be located in front of this structure and to depolarize its synchrotron emission, they claim that the "Fan Region" is indeed an enormous object extending up to the Perseus Arm, i.e. up to $\approx \mathrm{kpc}$ distance.

Another common and remarkable characteristic of the selected areas is that the polarization vectors appear mostly aligned at the two higher frequencies of the Leiden surveys.

\section{Map production algorithm and consistency tests}

A simple method to project the Leiden surveys into HEALPix maps is to average the observations falling in each pixel. However, the maps of the polarized intensity $(P I)$ and of the Stokes parameters $(Q, U)$ produced in this way show discontinuities on scales $\theta \simeq \theta_{\text {pixel }}$. These discontinuities tend to add spurious power in the recovered APS, particularly at multipoles $\ell \sim 180 / \theta_{\text {pix }}$. In order to smooth these discontinuities we implemented a "class" of specific "interpolation" algorithms to generate maps with $\theta_{\text {pix }} \sim 0.92^{\circ}$, for the whole sky region covered by the surveys. To each pixel is assigned a weighted average of the signal values falling in its neighbourhood, i.e. $\sum_{i}\left(p_{i} \cdot x_{i}\right) / \sum p_{i}$ with $p_{i}=1 /\left(\sigma_{i}^{2} \cdot d_{i}^{\mathrm{n}}\right)$, where $x_{i}$ and $\sigma_{i}$ are the signal and the error associated with the observation and $d_{i}$ its angular distance from the pixel centre. The radius within which the average is computed varies pixel by pixel and is selected according to the following guidelines: (i) to have enough observations (at least 3); (ii) to use only observations quite close to the considered pixel centre (less than a few degrees); (iii) to minimize the resulting average fractional change of the signal with the variation of the number of points and of the radius. The first two conditions imply that the interpolation is as local as possible and is performed using a reasonable number of data points. The third recipe is a convergency criterion.

In order to check the reliability of the method, we generated at each frequency several groups of $P I, Q$, and $U$ maps, each corresponding to a different choice of the distance power in the average weight $\left(1 /\left(\sigma^{2} \cdot d^{\mathrm{n}}\right)\right)$ for various pixel sizes (ranging between $3.7^{\circ}$ and $0.92^{\circ}$ ). A detailed description of the consistency tests together with preliminary results referring to a first version of the polarized intensity maps have been reported in La Porta (2001) and Burigana \& La Porta (2002). In that work we produced simulated maps of white noise, expected to contamine the astrophysical signal contained in the maps, according to three different recipes. We compared the APS of the simulated noise and signal maps to identify the ranges of multipoles statistically relevant (i.e. where the signal is not masked by the noise) for various considered sky coverages. We found that the analysis of the survey full coverage provides a good estimate of the polarization APS for $\ell \sim[2,50]$, while the better sampled patches (characterized by a higher $\mathrm{S} / \mathrm{N}$ ratio on average) allow us to investigate the multipole interval $\sim[30,100]$. We emphasize that the current APS analysis has a statistical meaning and cannot provide details on the local properties of the synchrotron emission.

\section{Interpolation algorithm optimization and accuracy}

In order to evaluate and optimize the signal reconstruction quality provided by our algorithm, we performed a final test. Starting from simulated polarization maps having known statistical properties, we created a table of data analogous to the Leiden survey ones and run our code to build $P I, Q$ and $U$ maps to be compared with the input skies. We focused on the $1411 \mathrm{MHz}$ case, because this is the most seriously undersampled both in terms of number of observations per beam and of observed points. Therefore, the maps produced at this frequency are potentially most influenced by the interpolation method effects, particularly at multipoles larger than some tens. We assumed $C_{\ell}^{E}=C_{\ell}^{B}=C_{\ell}=\kappa \cdot \ell^{\alpha}$, with values of the parameters $\kappa$ and $\alpha$ in agreement with the mean law obtained for the Galactic polarized synchrotron emission in our first analysis of the $1411 \mathrm{MHz}$ survey (reported in Burigana $\&$ La Porta 2002). For $\ell \sim[30,200]$ we adopted ${ }^{7} \kappa=1\left(K^{2}\right)$ and $\alpha=-3$. Applying the HEALPix facility SYNFAST to these angular power spectra, we generated maps of $Q$ and $U$ with a pixel size of $\simeq 13.7^{\prime}$ and a beamwidth $\theta_{\mathrm{HPBW}}=36^{\prime}$, as in the $1411 \mathrm{MHz}$ survey, and used them to build the simulated table of data as described below.

At each observed position of the Leiden survey

a) we first assigned the Stokes parameters corresponding to the SYNFAST simulation pixel in which it would project; and

b) the corresponding rms given in the original table;

c) we then added to each Stokes parameter a random value extracted from a Gaussian distribution in order to mimic the effect of the instrumental white noise (see, e.g., Burigana \& Sáez 2003). The adopted standard deviation of this Gaussian distribution has been chosen equal to the mean error quoted for the considered original data. In this way the signal-tonoise ratio of the simulated table is statistically very close to the real case one.

We then obtained $P I$ as $\sqrt{Q^{2}+U^{2}}$ and calculated its error $\sigma_{P I}$ according to the standard error propagation rules.

Finally, we applied our interpolation method to the simulated data table and produced independently $P I, Q$, and $U$ simulated maps with $\theta_{\text {pix }} \simeq 0.92^{\circ}$. We ran our code several times, changing the weight $n$ to be adopted in the average and the criteria for selecting the interpolation radius, so to sort out the best solution.

As a first self-consistency test of the resulting maps, we verified that typically the $P I$ map can be considered equal to the $\sqrt{Q^{2}+U^{2}}$ within the uncertainty limit in $85 \%$ of the pixels. Then we compared the reconstructed maps with the input sky maps directly obtained with the SYNFAST facility (see Fig. 1).

We first degraded ${ }^{8}$ the SYNFAST simulations to $0.92^{\circ}$ pixel size maps and considered only the portion of the sky covered by the Leiden survey. Computing the APSs of both the original and the reprocessed maps we found that the best agreement between them is obtained:

1. adopting as polarized intensity the quantity $\sqrt{Q_{\text {reproc }}^{2}+U_{\text {reproc }}^{2}}$ (instead of the $P I_{\text {reproc }}$, produced applying the filling code to the $P I$ values of the data table);

2. using the weight $1 /\left(\sigma^{2} \cdot d^{4}\right)$; and

\footnotetext{
7 For lower multipoles we assumed a slope $\leq-2.5$. Precisely, in this test we used $\alpha=-2.5$ for $\ell \sim[6,30]$ and $\alpha=0$ for $\ell<6$.

8 We verified that this operation does not significantly affect the APS on the multipole range of interest.
} 

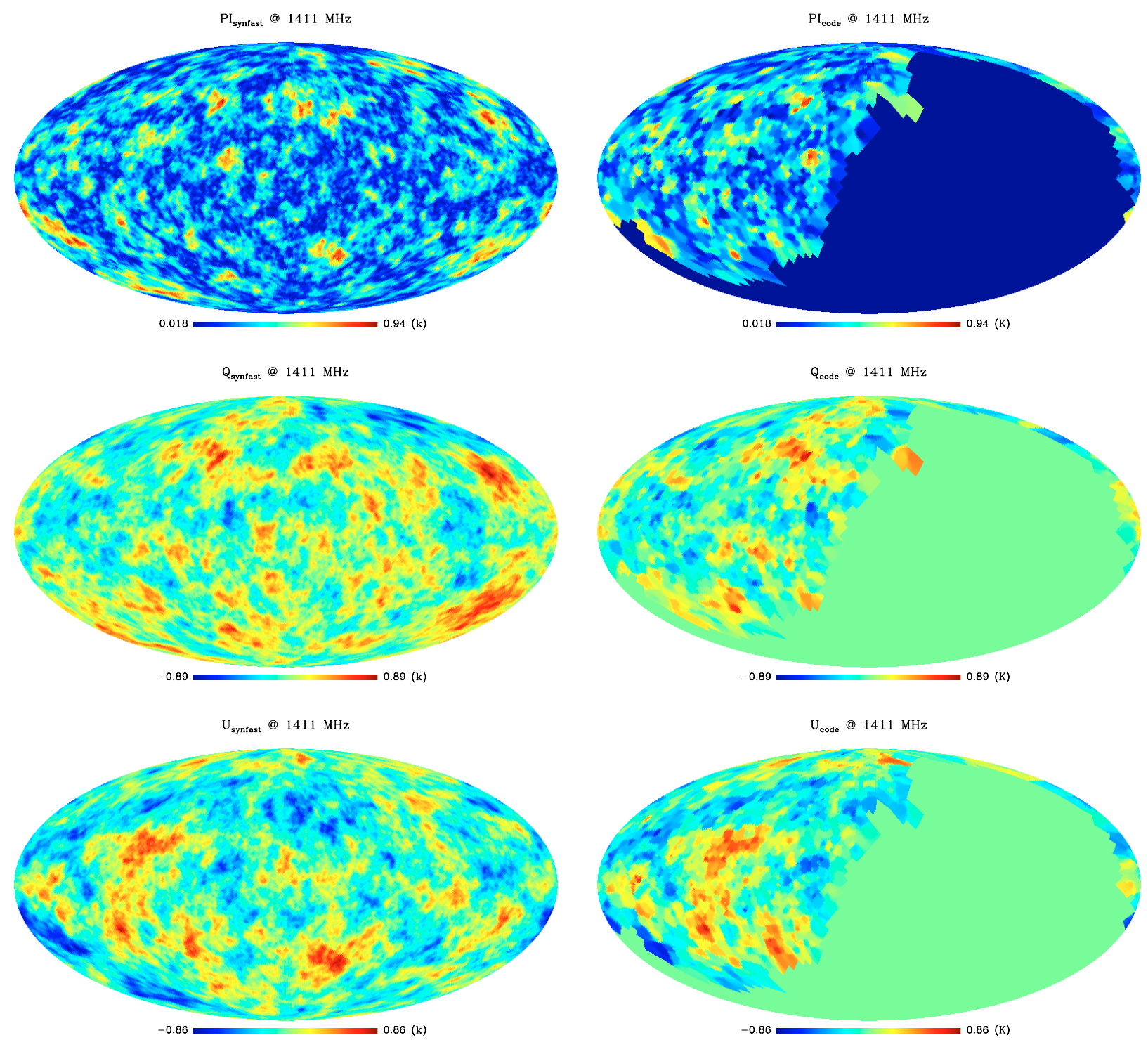

Fig. 1. SYNFAST realizations (left side) at $1411 \mathrm{MHz}$ versus maps reconstructed applying the final version of the filling code to the synthetic data table (right side). See text for details.

3. interpolating the data in the minimum circle containing at least two observations.

The first choice is motivated by the necessity to (implicitly) preserve the information on the polarization angle and is a posteriori justified by the following result: the APS of PI map reconstructed applying the code directly on the table of $P I$ values presents a certain loss in power with respect to the input case. The reason is likely that the $P I$ interpolated value overestimates the effective mean value of the field in the presence of changes in the signs of the Stokes parameters and consequently leads to an underestimation of the mean value of the field fluctuations.

Criteria 2 and 3 imply that the interpolation is as local as possible according to the sampling of each sky region. This approach is quite different from that pursued by other authors (Bruscoli et al. 2002); they performed a Gaussian smoothing everywhere with constant $\theta_{\text {HPBW }}$ (of $3^{\circ}$ ), neglecting the sampling dependence on the position in the sky and on the frequency.

In principle, before computing any average we should have transformed the polarization vectors using the formula of the parallel transport on the sphere; however operating only a local interpolation, the consequent mixing of the two orthogonal components of the polarized signal turned out to be negligible ${ }^{9}$.

Figure 2 shows that the statistical information contained in the synthetic data table is appreciably traced out in the

${ }^{9}$ In order to drag vectors on the sphere preserving strength and angles, one should apply the parallel transport formula estabilished by differential geometry. Integrating on paths close to geodetic, a suitable approximation is given by

$p_{\theta}^{(P T)}=p_{\theta} \cdot \cos (t)+p_{\phi} \cdot \sin (t)$

$p_{\phi}^{(P T)}=-p_{\theta} \cdot \sin (t)+p_{\phi} \cdot \cos (t)$

where $t=\cos \left(\theta_{\text {ave }}\right) \cdot \delta \phi$ and $\theta_{\text {ave }}=\left(\theta_{\text {in }}+\theta_{\text {fin }}\right) / 2$ (see Bruscoli et al. 2002 , for further details). Using these equations it is possible to estimate the percentage contribution of each initial Stokes parameter to the new parameter expression, once it has been moved to another point of the sphere. We assumed $\delta \phi=3^{\circ}$, corresponding to the mean value of the interpolation radius in the selected patches. Neglecting the parallel transport in our interpolation method causes a mixing of the Stokes parameters of $\simeq 1 \%$ in the equatorial area and of $\simeq 5 \%$ in the medium and high latitude regions. 
Table 1. The pixel to pixel difference between the maps directly generated with SYNFAST (after degradation to $0.92^{\circ}$ pixel size maps) and the reconstructed maps contaminated by white noise, for the whole survey and the considered patches. Columns 2 and 3 give the average and rms of the Gaussian function that best fits the difference distribution (shown in Fig. 3). Columns 4 and 5 contain the average and standard deviation of the difference distribution. Columns 6-8 give the percentages of pixels with absolute difference within the reported levels (here $1 \sigma$ corresponds to the rms reported in Col. 5).

\begin{tabular}{|c|c|c|c|c|c|c|c|}
\hline \multirow{2}{*}{ Half-sky } & \multicolumn{2}{|c|}{ Gaussian interp. } & \multicolumn{2}{|c|}{ Distribution } & \multicolumn{3}{|c|}{$\%$ of pixels } \\
\hline & ave $(\mathrm{mK})$ & $\mathrm{rms}(\mathrm{mk})$ & ave $(\mathrm{mK})$ & $\mathrm{rms}(\mathrm{mk})$ & $1 \sigma$ & $2 \sigma$ & $3 \sigma$ \\
\hline$\Delta P I$ & 3.6 & 88.3 & 3.9 & 102.2 & 71.8 & 94.7 & 99.0 \\
\hline$\Delta Q$ & -2.4 & 97.1 & -0.9 & 109.6 & 71.5 & 94.9 & 99.1 \\
\hline$\Delta U$ & -5.6 & 93.5 & -9.1 & 114.3 & 73.7 & 94.8 & 98.7 \\
\hline \multirow[t]{2}{*}{ Patch 1} & \multicolumn{2}{|c|}{ Gaussian interp. } & \multicolumn{2}{|c|}{ Distribution } & \multicolumn{3}{|c|}{$\%$ of pixels } \\
\hline & ave $(\mathrm{mK})$ & rms (mk) & ave $(\mathrm{mK})$ & $\mathrm{rms}(\mathrm{mk})$ & $1 \sigma$ & $2 \sigma$ & $3 \sigma$ \\
\hline$\Delta P$ & 6.3 & 61.6 & 7.7 & 73.7 & 71.8 & $\overline{94.4}$ & $\overline{98.9}$ \\
\hline$\Delta Q$ & -7.5 & 68.2 & -8.2 & 76.2 & 70.8 & 95.4 & 99.2 \\
\hline$\Delta U$ & -6.6 & 66.1 & -6.8 & 77.4 & 72.1 & 94.4 & 99.1 \\
\hline \multirow[t]{2}{*}{ Patch 2} & \multicolumn{2}{|c|}{ Gaussian interp. } & \multicolumn{2}{|c|}{ Distribution } & \multicolumn{3}{|c|}{$\%$ of pixels } \\
\hline & ave $(\mathrm{mK})$ & $\mathrm{rms}(\mathrm{mk})$ & ave $(\mathrm{mK})$ & $\mathrm{rms}(\mathrm{mk})$ & $1 \sigma$ & $2 \sigma$ & $3 \sigma$ \\
\hline$\Delta P I$ & 1.2 & 74.9 & 3.5 & 80.3 & 70.7 & $\overline{95.1}$ & $\overline{99.6}$ \\
\hline$\Delta Q$ & 1.6 & 81.1 & -3.5 & 85.5 & 70.0 & 94.8 & 99.4 \\
\hline$\Delta \widetilde{U}$ & -3.2 & 75.0 & -10.2 & 85.2 & 70.5 & 95.9 & 99.0 \\
\hline \multirow[t]{2}{*}{ Patch 3} & \multicolumn{2}{|c|}{ Gaussian interp. } & \multicolumn{2}{|c|}{ Distribution } & \multicolumn{3}{|c|}{$\%$ of pixels } \\
\hline & ave $(\mathrm{mK})$ & rms (mk) & ave $(\mathrm{mK})$ & $\mathrm{rms}(\mathrm{mk})$ & $1 \sigma$ & $2 \sigma$ & $3 \sigma$ \\
\hline$\Delta P I$ & 6.0 & 72.8 & 1.4 & 78.9 & 69.4 & 96.3 & 99.7 \\
\hline$\Delta Q$ & 1.2 & 68.5 & -3.4 & 71.9 & 69.0 & 94.6 & 99.7 \\
\hline$\Delta U$ & -13.9 & 75.7 & -13.9 & 81.0 & 68.0 & 94.6 & 99.3 \\
\hline
\end{tabular}

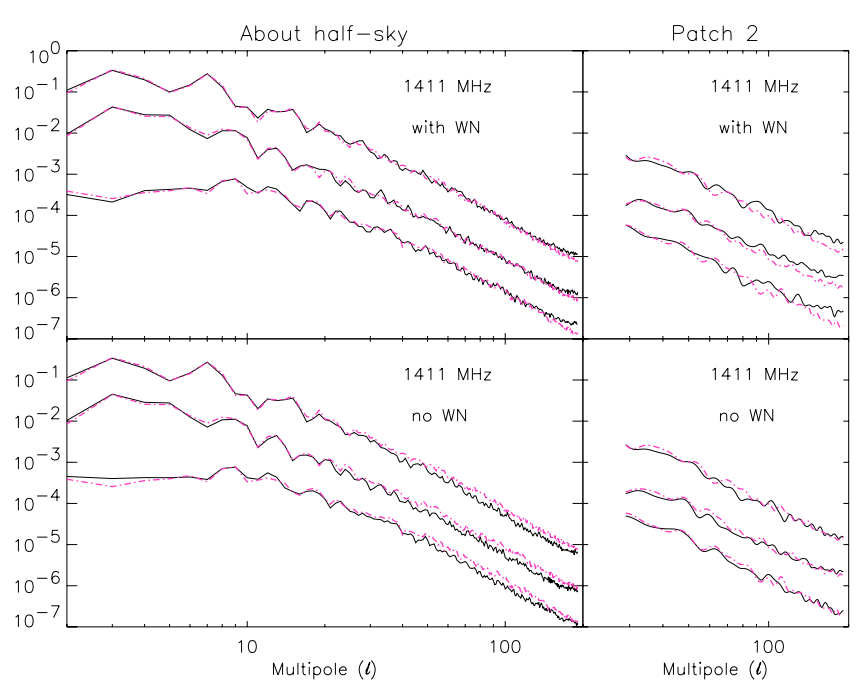

Fig. 2. Comparison between the APSs of the input maps (dot lines) extracted from the SYNFAST simulations (degraded to $0.92^{\circ}$ pixel size maps) and the reprocessed maps (solid lines). From the bottom: $C_{\ell}^{P I}$, $C_{\ell}^{E} \cdot 10$ and $C_{\ell}^{B} \cdot 100$. Note the excellent agreement between the input and reprocessed APSs up to $\sim 100$.

multipole ranges of interest both for the half-sky map and for the patches (for conciseness we report here only the results found for patch 2).

Fitting the APS of the reprocessed map, we quantified the precision level at which the input values of the amplitude and of the spectral index were recovered: by considering several realizations for the various cases, we found relative errors within $\simeq 10 \%$ for both the parameters.

We also checked the reliability of the maps themselves in a more quantitative way. The distributions of the pixel to pixel difference between the two series of maps (i.e. $P I, Q$ and $U$ produced by SYNFAST and reprocessed by our interpolation code) are, in all cases, close to Gaussian distributions, with standard deviations comparable with the typical mean error of the data table (see Table 1 and Fig. 3).

We repeated the test described above skipping the step c) ${ }^{10}$. The distributions of the differences between the input and reprocessed maps are again close to Gaussian distributions, but with standard deviations slightly smaller than those found including step c) (in particular, for the full survey coverage the standard deviation is now smaller by about $20 \%$ ). This indicates the existence of a geometric contribution in the error distribution over the maps related to the poor sampling of the data. Expressing the rms of the pixel to pixel difference distribution as

$\sigma_{\text {map }} \simeq \sqrt{\sigma_{\text {samp }}^{2}+\sigma_{\mathrm{WN}}^{2}}$

the analysis of reprocessed maps (with and without noise) gives

$\sigma_{\mathrm{WN}} \simeq$ const. $\times \sigma_{\text {samp }}$

with const. $\simeq 0.63,0.87,0.90,1.15$, respectively for the whole survey coverage and for the patches 1,2 , and 3, without significant differences among the maps of $P I, Q$ or $U$.

The relative weight of the geometrical contribution to the overall error decreases with the goodness of the sampling. At the other frequencies of the Leiden surveys the sampling and the signal-to-noise ratio are better than at $1411 \mathrm{MHz}$; as a consequence, we can look at the ratio between $\sigma_{\text {samp }}$ (resp. $\sigma_{\text {map }}$ ) and the signal at $1411 \mathrm{MHz}$ as a conservative upper limit to the relative error caused by geometric effects (resp. geometric effects plus experiment white noise) at the lower frequencies.

To verify whether the goodness of the signal reconstruction depends on the input sky characteristics, we repeated the entire test adopting a different input APS, more appropriate at $408 \mathrm{MHz}$. At this frequency the analysis of the preliminary polarization maps led to different results for the APSs than

\footnotetext{
10 Formally, in this case we apply the step c) but using in the noise generation a standard deviation negligible with respect to the values of $P I, Q$ and $U$ (the implemented code assumes that a mean error is given for each measurement of the observed quantities).
} 


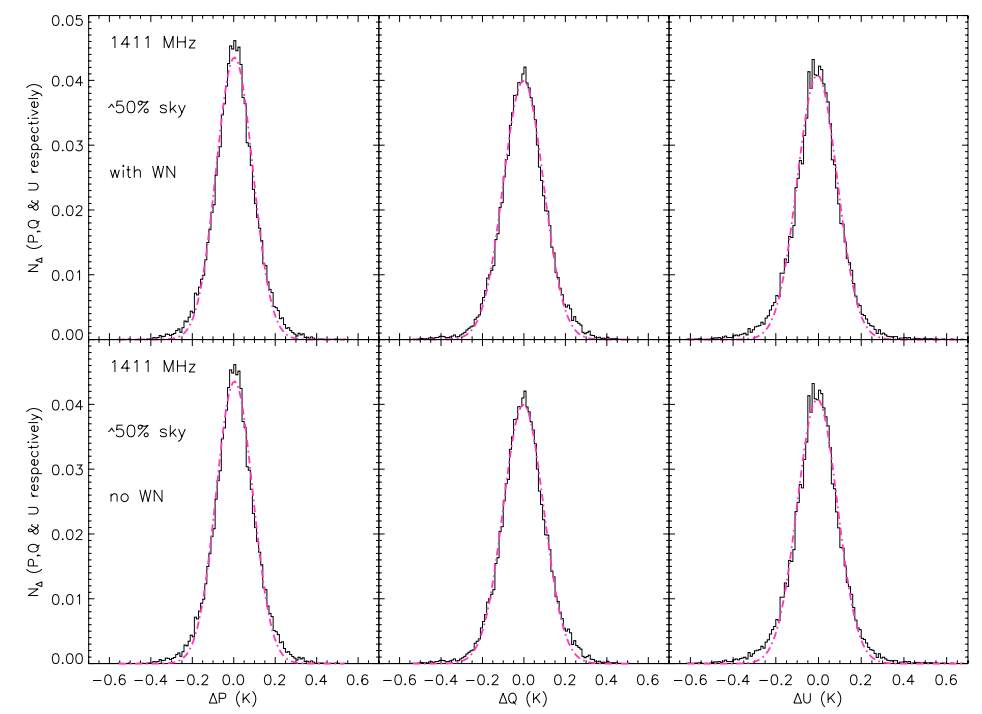

Fig. 3. Distribution of the pixel to pixel differences between the maps directly simulated with SYNFAST (degraded to $0.92^{\circ}$ pixel size maps) and the reprocessed maps at $1411 \mathrm{MHz}$. We display here the results of the test, both with (upper panels) and without (lower panels) white noise contamination, in the case of the full coverage $1411 \mathrm{MHz}$ Leiden survey. The superimposed curves are the Gaussian distributions giving the best fit to the data.

the $1411 \mathrm{MHz}$ case. In particular, the spectral index was flatter (roughly $\sim-1.5$ in the multipole range [30, 200]), both for the whole coverage map and for the patches. The same test repeated with this slope confirmed the results described before. The quality of the APS reconstruction is slightly less accurate than in the previous case, however the relative errors estimated for the recovered amplitudes and spectral indices remain within $\simeq 10 \%$.

\section{Results}

In Fig. 4 we show the polarization maps $(P I, Q$, and $U$ ) produced at all frequencies with the final version of the code described in the previous section.

Using the ANAFAST facility of the HEALPix package, we computed the APSs of the polarized intensity and of the $E$ and $B$ modes for the half-sky coverage and for the patches ${ }^{11}$ at the five frequencies of the surveys. In addition to the three better sampled areas we considered another region (patch 4) located at $\left(70^{\circ} \leq l \leq 120^{\circ},-45^{\circ} \leq b \leq-15^{\circ}\right)$. This patch has a sky sampling in the average (its APS is then statistically relevant only for $\ell \lesssim 50$ ) and is characterized by a rather low signal.

The $E$ and $B$ modes turned out to be extremely similar, so that, instead of studying each of them singularly, we consider their average $\left(C_{\ell}^{E}+C_{\ell}^{B}\right) / 2$. In some cases (namely for patch 1 and 2 at $1411 \mathrm{MHz}$ and patch 2 and 4 at $408 \mathrm{MHz}$ ) we could even consider a unique APS defined as $\left(C_{\ell}^{P I}+C_{\ell}^{E}+C_{\ell}^{E}\right) / 3$.

The maps represent the Galactic polarized synchrotron emission, smoothed with the beam of the radiotelescope and contaminated by the noise. As a consequence, their APSs can be fit as sum of two components, $C_{\ell}=C_{\ell}^{\text {synch }} W_{\ell}+C_{\ell}^{\mathrm{N}}$. We exploit the power law approximation $C_{\ell}^{\text {synch }} \simeq \kappa \cdot \ell^{\alpha}$ and assume a symmetric, Gaussian beam, i.e. a window function $W_{\ell}=\mathrm{e}^{-\left(\sigma_{b} \ell\right)^{2}}$, where $\sigma_{b}=\theta_{\mathrm{HPBW}}(\mathrm{rad}) / \sqrt{8 \ln 2}$. Under the hypothesis of uncorrelated Gaussian random noise ${ }^{12}$ (white noise), $C_{\ell}^{\mathrm{N}}=C_{\ell}^{\mathrm{WN}} \sim$ const.

11 The monopole of each map has been subtracted before APS estimation and the obtained $C_{\ell}$ have been divided by the fractional coverage of the considered area to renormalize it to the whole sky case.

${ }_{12}$ Even in the ideal case of negligible systematic effects in the data, the averaging and the interpolation over the poorly sampled data may
We have performed the fit on the multipole range [30,200] for the patches and $[2,200]$ for the full-coverage maps. In fact, the flattening occurring at higher multipoles helps recover the noise constant, in spite of the fact that no reliable astrophysical information can be derived for $\ell>100$ from the APSs even in the three better sampled regions (Burigana \& La Porta 2002). For the patches, we found that a single set of the parameters $\kappa, \alpha$ allows us to describe the APS in the entire range of multipoles. In the case of the survey full coverage we needed two sets of $\kappa$, $\alpha$, each of them appropriate to a certain multipole range. At all frequencies a change in the APS slope occurs corresponding to $\ell \sim 10$ for both $C_{\ell}^{P I}$ and $\left(C_{\ell}^{E}+C_{\ell}^{B}\right) / 2$.

As an example, we display in Fig. 5 the APS and the corresponding best fit curve at $610 \mathrm{MHz}$ for the survey full coverage and one patch. We show in Fig. 6 (resp. Fig. 7) the recovered synchrotron term, $C_{\ell}^{\text {synch }}$, and list in Table 2 (resp. Table 3 ) the corresponding $\kappa, \alpha$ obtained for the survey full coverage (resp. for the patches). In the multipole range $30 \lesssim \ell \lessgtr 100$ we found a general trend: from the lowest to the highest frequency the slope increases from $\sim-(1-1.5)$ to $\sim-(2-3)$, with a weak dependence on the considered sky region.

\section{Comparison with the DRAO polarization survey}

Soon, a new all-sky survey at $1.4 \mathrm{GHz}$ will become available. It results from the merging of two different polarization surveys having the same angular resolution of about $36^{\prime}$ : the Northern part of the sky has been observed with the DRAO $26 \mathrm{~m}$ telescope (Wolleben et al. 2003), for the Southern hemisphere the $30 \mathrm{~m}$ Villa Elisa telescope was used (Testori et al. 2003). The first was meant to compensate for the lack of information typical of the Leiden surveys and it improves their sampling and sensitivity. However the Leiden surveys remain so far a unique tool for absolute calibration in the Northern sky, therefore the DRAO data have been tightened to them. A second observing

imply deviations from this simple assumption. Moreover, the noise level in the maps will be connected not only to the instrumental noise, but also to the sampling. A posteriori, the APS flattening found at high $\ell$ justifies our simple approximation. 

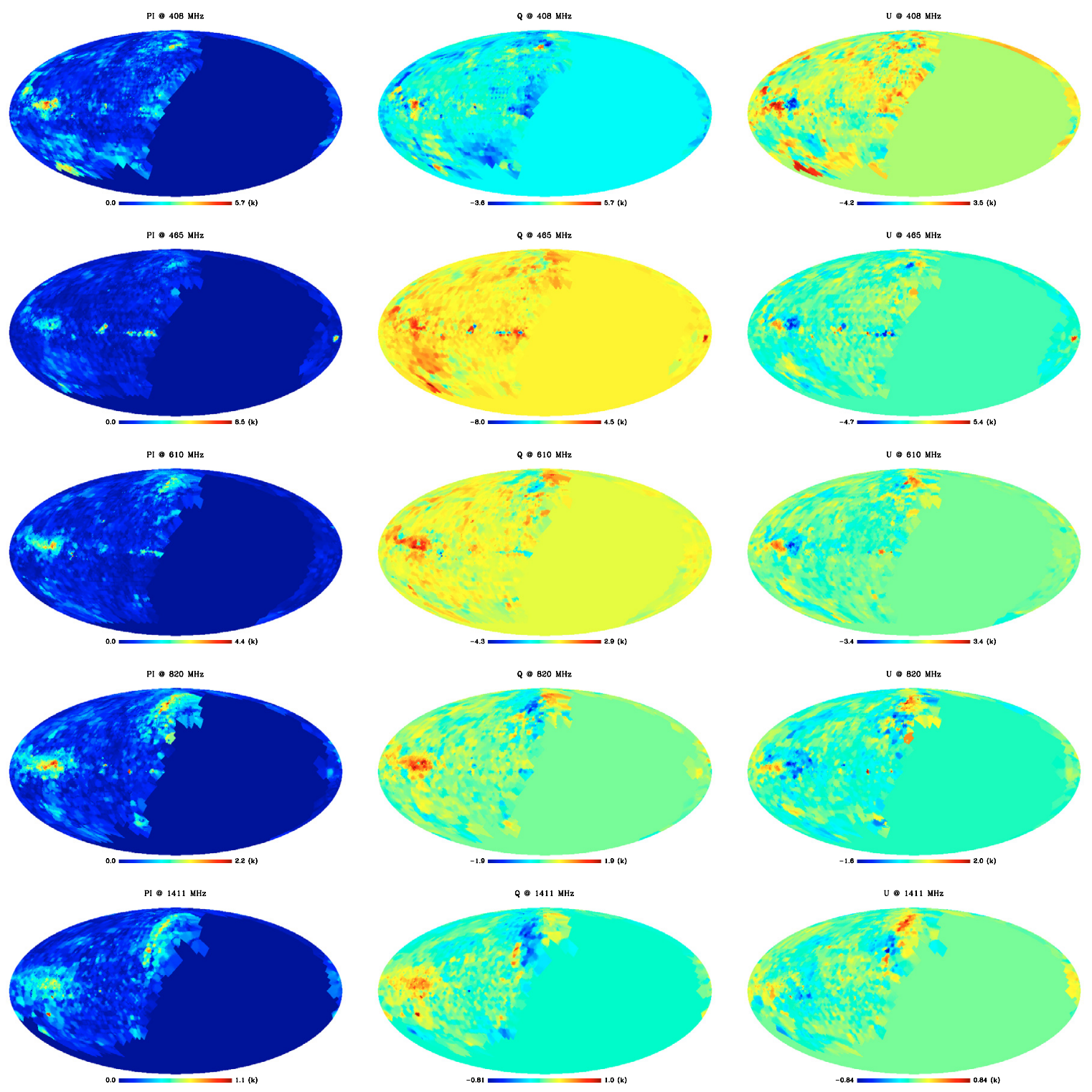

Fig. 4. $P I, Q$, and $U$ maps obtained from the Leiden surveys data tables using the final version of the interpolation algorithm at all frequencies.

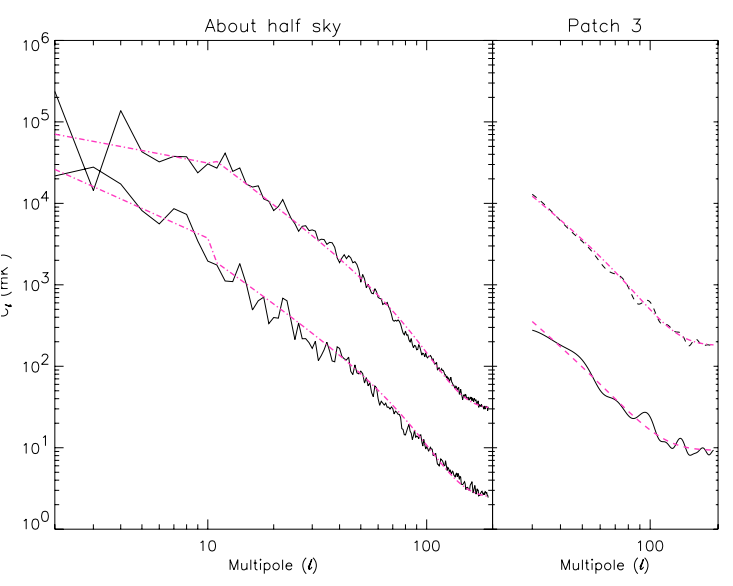

Fig. 5. Polarization APSs at $610 \mathrm{MHz}$ (solid lines) for the survey full coverage (left panel) and patch 3 (right panel) together with the corresponding best fit curves (dot-dash lines). The lower curves in each panel are $C_{\ell}^{P I}$, while the other ones represent $\left(C_{\ell}^{E}+C_{\ell}^{B}\right) / 2$ multiplied by 10 .

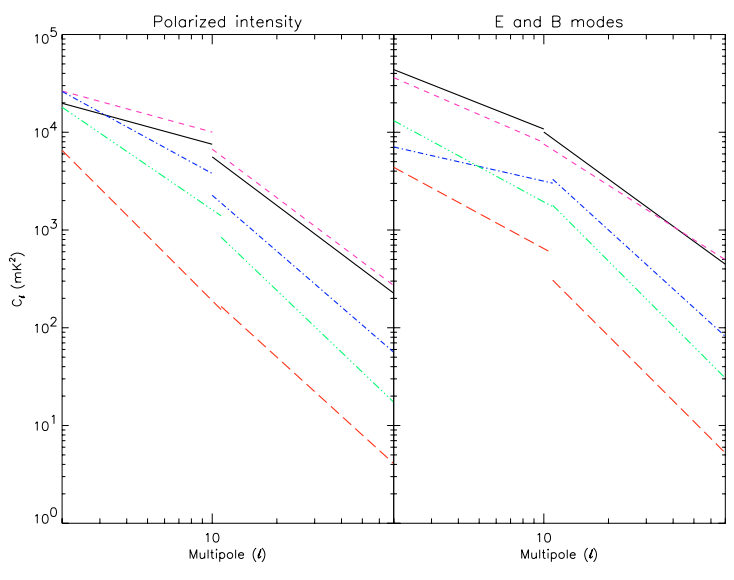

Fig. 6. Synchrotron component of the APS derived for the survey full coverage for the polarized intensity (left panel) and for $\left(C_{\ell}^{E}+C_{\ell}^{B}\right) / 2$ (right panel) at the various frequencies: 408 (solid line), 465 (dashes), 610 (dot-dash), 820 (three dot-dash), and $1411 \mathrm{MHz}$ (long dashes). 


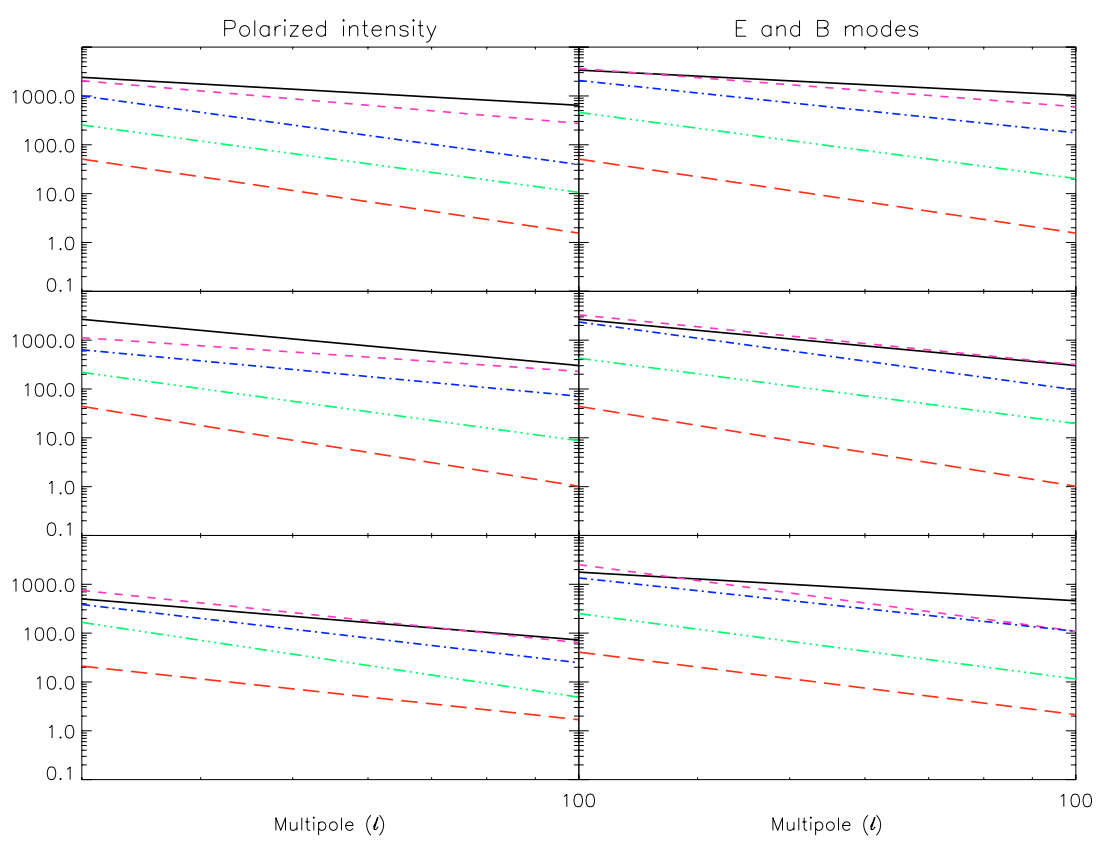

Fig. 7. As in Fig. 6 but for the selected patches: patch 1 (top panels), patch 2 (middle panels), and patch 3 (bottom panels).

Table 2. Values of the best fit parameters $\kappa$ and $\alpha$ obtained in the analysis of the survey full coverage APS at all the frequencies (as shown in Fig. 6). Relative errors on the best fit parameters are $\sim 10 \%$. Two multipole ranges are considered: $[2, \ell]$ and $[\ell, 200]$ ( $\ell$ was also a free parameter in the fit). $E B$ refers to $\left(C_{\ell}^{E}+C_{\ell}^{B}\right) / 2$.

\begin{tabular}{ccccccc}
\hline \hline $\begin{array}{c}v \\
(\mathrm{MHz})\end{array}$ & $\begin{array}{c}C_{\ell} \\
\left(\mathrm{mK}^{2}\right)\end{array}$ & $\begin{array}{c}\kappa_{1} \\
\left(\mathrm{mK}^{2}\right)\end{array}$ & $\alpha_{1}$ & $\ell$ & $\begin{array}{c}\kappa_{2} \\
\left(\mathrm{mK}^{2}\right)\end{array}$ & $\alpha_{2}$ \\
\hline 408 & $E B$ & $8.00 \times 10^{4}$ & -0.87 & 10.86 & $4.00 \times 10^{5}$ & -1.60 \\
& $P I$ & $3.00 \times 10^{4}$ & -0.60 & 10.80 & $2.50 \times 10^{5}$ & -1.65 \\
\hline 465 & $E B$ & $7.00 \times 10^{4}$ & -0.95 & 10.00 & $1.90 \times 10^{5}$ & -1.40 \\
& $P I$ & $4.00 \times 10^{4}$ & -0.60 & 10.85 & $3.00 \times 10^{5}$ & -1.65 \\
\hline 610 & $E B$ & $1.00 \times 0^{4}$ & -0.50 & 10.00 & $4.00 \times 10^{5}$ & -2.00 \\
& $P I$ & $6.00 \times 0^{4}$ & -1.20 & 10.85 & $1.8 \times 10^{5}$ & -1.90 \\
\hline 820 & $E B$ & $3.00 \times 0^{4}$ & -1.19 & 11.00 & $3.5 \times 10^{5}$ & -2.20 \\
& $P I$ & $5.10 \times 0^{4}$ & -1.50 & 11.00 & $1.30 \times 10^{5}$ & -2.10 \\
\hline 1411 & $E B$ & $1.00 \times 10^{4}$ & -1.19 & 11.00 & $6.00 \times 10^{4}$ & -2.20 \\
& $P I$ & $3.00 \times 10^{4}$ & -2.20 & 11.00 & $2.00 \times 10^{4}$ & -2.00 \\
\hline & & & & & &
\end{tabular}

period at DRAO has recently been completed. The final map is fully sampled in right ascension and has a spacing in declination ranging between $0.25^{\circ}$ and $2.5^{\circ}$ (Wolleben et al. 2006). In Fig. 8 we compare the APS of $C_{\ell}^{P I}, C_{\ell}^{E}$, and $C_{\ell}^{B}$ derived from a preliminary version of the DRAO survey with the ones of the maps we constructed using the Leiden survey at $1411 \mathrm{MHz}$. For the full coverage maps the APSs almost coincide at $\ell \lesssim 10$. At $\ell \gtrsim$ few $\times 10$ the slopes are in good agreement in all cases, while the amplitudes derived from the Leiden survey are larger by a factor $\sim 2.5$ for the full-coverage maps and $\sim 4$ for the patches. As a consequence, the fitted amplitudes reported in the previous section might translate in an overestimate of the synchrotron emission APS at $1.4 \mathrm{GHz}$. We checked that the signal in the DRAO survey is typically lower than in map we constructed from the Leiden survey at $1411 \mathrm{MHz}$, which seems consistent with the amplitude discrepancy found. A detailed analysis of the final version of the recent $1.4 \mathrm{GHz}$ polarization data and its comparison with the Leiden $1.4 \mathrm{GHz}$ survey is in progress. We stress here the good agreement between the slopes of the APSs obtained from the two data sets that confirms the steepness of the APS we found at $1.4 \mathrm{GHz}$ from the Leiden survey.

\section{Multifrequency analysis}

In Sect. 5 we studied the APS of the Galactic diffuse polarized emission at each frequency, focusing on its multipole dependence. We first consider here the behaviour of the synchrotron APS at some fixed values of the multipole as function of frequency, being aware of the depolarization phenomena. In the second subsection we provide a qualitative interpretation of the APS steepening with increasing frequency in terms of Faraday depolarization effects.

\subsection{Frequency dependence of the APS amplitude and Faraday depolarization}

The theoretical intrinsic behaviour of the synchrotron total intensity emission predicts a power law dependence of the APS amplitude on frequency:

$T^{\text {synch }} \propto v^{-\beta-2} \Rightarrow C_{\ell}^{\text {synch }}(v) \propto v^{2(-\beta-2)}$.

The values of $\beta$ deduced from radio observations change with the considered sky position and range between $\sim 0.5$ and $\sim 1$ (see Reich \& Reich 1988; Platania et al. 1998). In principle, one would expect the synchrotron emission to be highly polarized, up to a maximum of $\sim 75 \%$ (see Ginzburg \& Syrovatskii 1965). In the ideal case of constant degree of polarization the above formulae would apply also to the polarized component of the synchrotron emission, once the brightness temperature has been properly rescaled. However, depolarization effects are relevant at radio frequencies, so that the predicted correlation between total and polarized intensity do not show up at all (except for the NPS and the Fan Region that are clearly evident both in total intensity and polarization maps at about $1.4 \mathrm{GHz}$ ). The current knowledge of $\beta$ for the Galactic diffuse polarized emission is very poor, but already indicates that due to depolarization phenomena the observed $\beta$ can be much lower than $\sim 0.7$ (e.g. for the NCP Vinyajkin \& Razin 2002 quote $\beta \sim-0.13$ ). 
Table 3. Values of best fit parameters $\kappa$ and $\alpha$ obtained in the analysis of the APS of the patches at all the frequencies (as shown in Fig. 7). Relative errors on the best fit parameters are $\sim 10 \%$. The considered multipole range is [30-200]. $E B$ refers to $\left(C_{\ell}^{E}+C_{\ell}^{B}\right) / 2$.

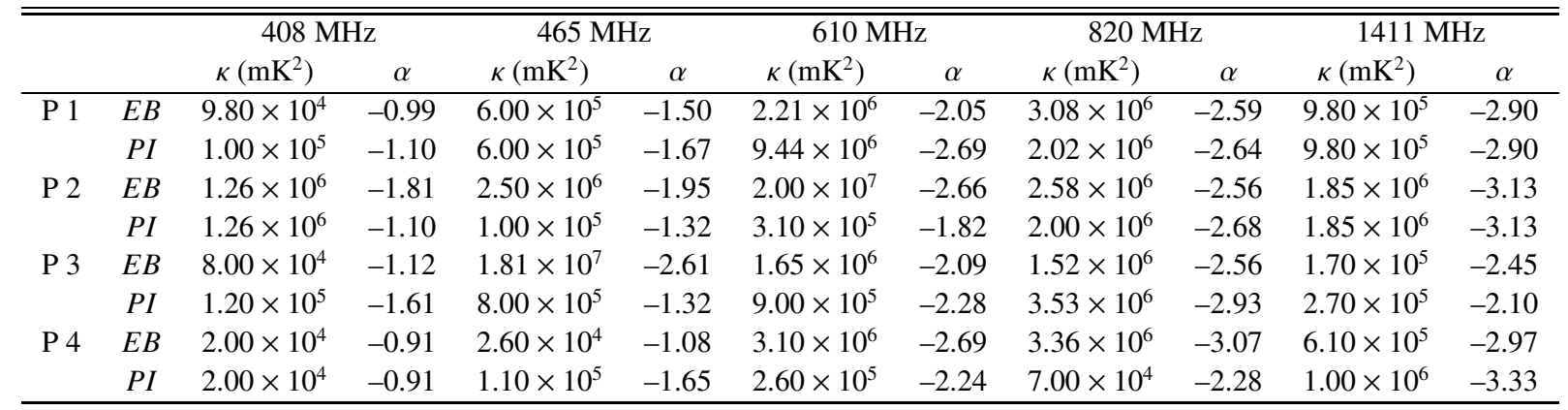
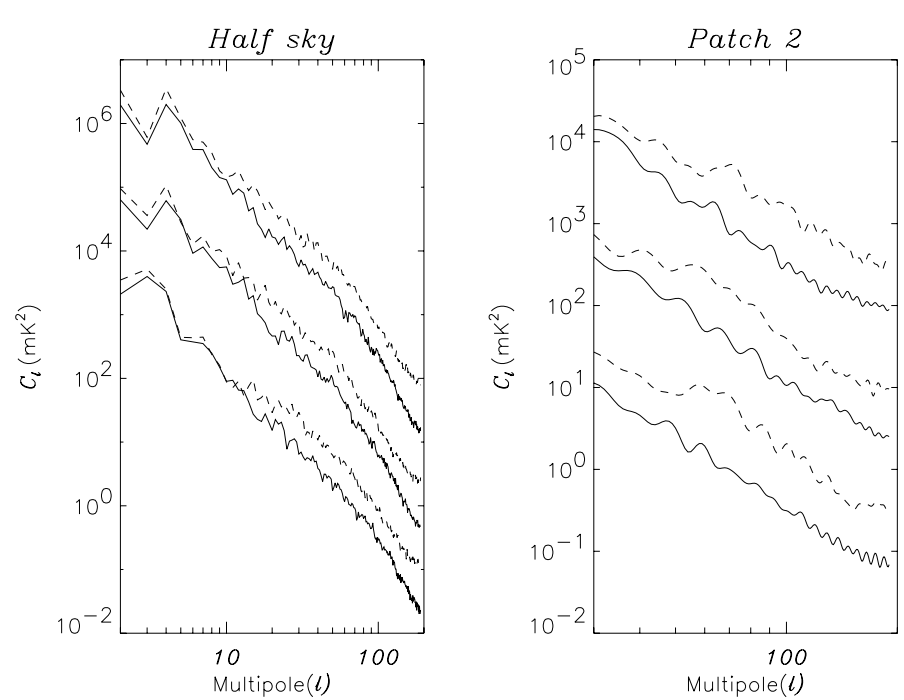

Fig. 8. Comparison between the DRAO APSs (solid lines) and the APSs of the maps reconstructed by our code (dashes) from the $1411 \mathrm{MHz}$ Leiden survey properly rescaled according to Wolleben (2005). In each panel, the three pairs of curves (from the bottom to the top) correspond to the $P I, E$, and $B$ modes; the $E$ and $B$ APSs have been multiplied respectively by 20 and 700 .

In Fig. 9 we plot $C_{\ell=\tilde{\ell}}^{\text {synch }}(v)$ at boundary and intermediate values of the statistically significant multipole range in each coverage case. For the patches we chose $\tilde{\ell}=30,50,70,100$, whereas for the half-sky maps we considered $\tilde{\ell}=5,30,50,70$. The APS amplitude actually decreases with frequency, but our results are not well fitted by a power law and exhibit shapes significantly flatter than that of the observed synchrotron emission in total intensity (approximately $C_{\ell}^{\text {synch }}(v) \propto v^{-5.4}$ ), as expected in the presence of frequency-dependent depolarization effects.

An electromagnetic wave travelling through a magnetized plasma will undergo a change in the polarization status. In particular its polarization vector will be rotated by an angle

$\Delta \phi[\mathrm{rad}]=R M\left[\mathrm{rad} / \mathrm{m}^{2}\right] \cdot \lambda^{2}\left[\mathrm{~m}^{2}\right]$.

Here the rotation measure, $R M$, is the line of sight integral

$R M\left[\mathrm{rad} / \mathrm{m}^{2}\right]=0.81 \int n_{\mathrm{e}}\left[\mathrm{cm}^{-3}\right] \cdot B_{\|}[\mu \mathrm{G}] \mathrm{d} l[\mathrm{pc}]$,

where $n_{\mathrm{e}}$ is the electron density and $B_{\|}$is the component of magnetic field along the line of sight.

Given $\Delta \phi \propto v^{-2}$, the lower the frequency the greater the importance of the Faraday rotation effect. Approximating the interstellar medium (ISM) as a superposition of infinitesimal layers
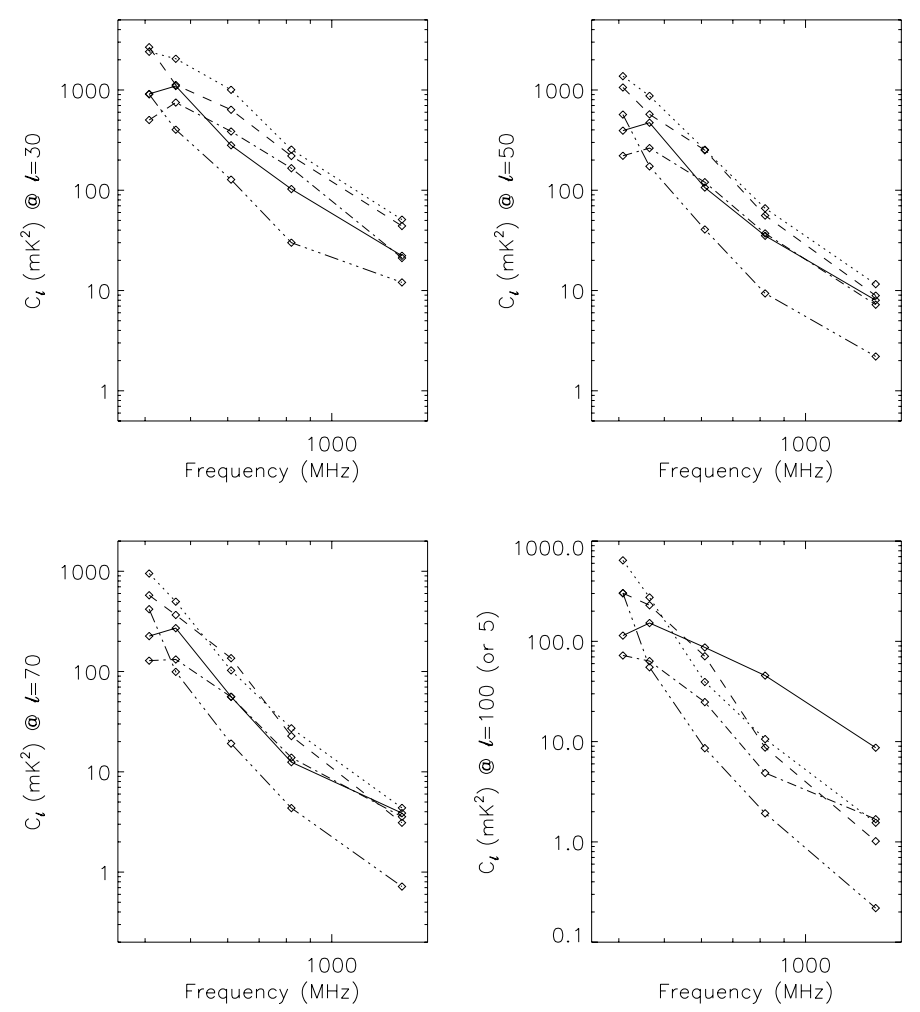

Fig. 9. Synchrotron PI APS at all frequencies for some fixed representative multipoles, $\bar{\ell}$ close to the boundary and in the middle of the range of validity of our analysis. We considered $\ell=30,50,70,100$ for the patches and $\ell=5,30,50,70$ for the full coverage maps. The diamonds represent the $C_{\bar{\rho}}^{\text {synch }}(v)$ at the survey frequencies. Different lines correspond to different sky regions, namely: full survey coverages (solid line), patch 1 (dots), patch 2 (dashes), patch 3 (dot-dashes), patch 4 (three dots-dashes).

each characterized by constant $n_{\mathrm{e}}$ and $B_{\|}$(slab model, see Burn 1966), the observed polarization intensity relates to the intrinsic one according to the expression:

$T^{\mathrm{p}, \mathrm{obs}}(\lambda)=T^{\mathrm{p}, \mathrm{intr}}(\lambda) \cdot|\sin \Delta \phi / \Delta \phi|$.

Therefore the net effect of a differential rotation of the polarization angle is a depletion of the polarized intensity, commonly referred to as Faraday depolarization. Given $\left(T^{\mathrm{p}}\right)^{2} \propto C_{\ell}(v)$ the above formula reads:

$C_{\ell}(v)=C_{\ell}^{\mathrm{intr}}(v) \cdot(\sin \Delta \phi / \Delta \phi)^{2}$ 
where $C_{\ell}^{\text {intr }}(v)=\kappa \cdot \ell^{\alpha} \cdot v^{2(-\beta-2)}$. At a given multipole, the ratio between the APSs at two frequencies can be written as:

$C_{\ell}\left(v_{1}\right) / C_{\ell}\left(v_{2}\right)=\left(v_{1} / v_{2}\right)^{-2 \beta-4} \cdot\left(\Delta \phi_{2} / \Delta \phi_{1}\right)^{2} \cdot\left(\sin \Delta \phi_{1} / \sin \Delta \phi_{2}\right)^{2}$.

Given a certain value of $\beta$, the expression on the right side of this equation can be displayed as a function of $R M$ (see Fig. 10). The $R M$ intervals in which the curve (obtained for a certain $\beta$ ) intersects the constant line representing the observed value of this ratio provide information about the possible $R M$ values $^{13}$. This holds in the case of pure Faraday depolarization. In reality there are at least two other phenomena affecting the observed polarized intensity, the beamwidth and the bandwidth depolarization (see Gardner \& Whiteoak 1966; and Sokoloff et al. 1998). If the polarization angles vary within the beam area, then observations of the same sky region with different angular resolution will give an apparent change of the polarized intensity with frequency (beamwidth depolarization). Suppose $\theta_{\mathrm{HPBW}, 1}$ and $\theta_{\mathrm{HPBW}, 2}\left(<\theta_{\mathrm{HPBW}, 1}\right)$ are the angular resolutions respectively at $v_{1}$ and $v_{2}$. The effect can be removed by smoothing the map at the frequency $v_{2}$ to the (lower) angular resolution of the map at the frequency $v_{1}$ or, equivalently, multiplying the observed APSs ratio, $C_{\ell}\left(v_{1}\right) / C_{\ell}\left(v_{2}\right)$, by $\mathrm{e}^{\left(\Delta \sigma_{b} \ell\right)^{2}}$, where $\Delta \sigma_{b}=\sigma_{1}{ }^{2}-\sigma_{2}{ }^{2}$ $\left(\sigma_{i}=\theta_{\mathrm{HPBW}, i}(\mathrm{rad}) / \sqrt{8 \ln 2} ; i=1,2\right)$. This correction ${ }^{14}$ has been applied to convert the APSs shown in Fig. 9 to the ratios shown in Fig. 10.

Another depolarization effect is introduced by the receiver bandwidth: the polarization angle will change by an amount

$\Delta \psi=-R M \cdot \lambda_{0}^{2} \cdot 2 \Delta v / v_{0}$,

where $\lambda_{0}$ and $v_{0}$ are the values at the bandwidth centre. The polarization of the incoming radiation will be reduced by a factor $\sin \Delta \psi / \Delta \psi$, that in our case is negligible $(\Delta v=1.7 \mathrm{MHz}$ at the two lower frequencies, $\Delta v=3.6 \mathrm{MHz}$ at the intermediate ones and $\Delta v=7.2 \mathrm{MHz}$ at the highest one).

In Fig. 10 we plot the $C_{\tilde{\ell}}^{\text {synch }}\left(v_{1}\right) / C_{\tilde{\ell}}^{\text {synch }}\left(v_{2}\right)$ at $\tilde{\ell}=30,70$ (resp. at $\tilde{\ell}=5,30$ ) in the case of the patches (resp. in the case of the full-coverage maps).

The information coming from the lower frequencies is too poor to be able to learn something about $R M \mathrm{~s}$; on the other hand, the ratio between the APSs at 820 and $1411 \mathrm{MHz}$ give some indications. For a reasonable range of synchrotron emission frequency spectral indices, the following values are compatible with the data (see left-bottom panel of Fig. 10): the relatively spread intervals $9-17,53-60,75-87,123-130 \mathrm{rad} / \mathrm{m}^{2}$ together with two narrow intervals at 70 and $140 \mathrm{rad} / \mathrm{m}^{2}$. In general there is a rather good agreement among the results coming from the patches and the full-coverage map. Spoelstra (1984) reported as a typical value $R M=8 \mathrm{rad} / \mathrm{m}^{2}$, however this is likely a lower limit to the real values (in addition, beam depolarization effects were not taken into account in that study, likely implying a certain underestimation of $R M$ ).

Another constraint to the possible values of the $R M s$ comes from the polarization degree, defined as

$\Pi=T^{\mathrm{p}} / T$.

\footnotetext{
${ }^{13}$ Clearly, the $R M$ values identified with this method do not refer to a precise direction in the sky, but to the considered sky area.

14 Once the $1411 \mathrm{MHz}$ map is properly smoothed to the lower resolution of the $820 \mathrm{MHz}$ survey, the bulk of possible differencies due to beam depolarization effects is eliminated and no longer contributes to the value of the ratio between the APSs at the two frequencies. Consequently, beam depolarization is not an issue in the following analysis.
}
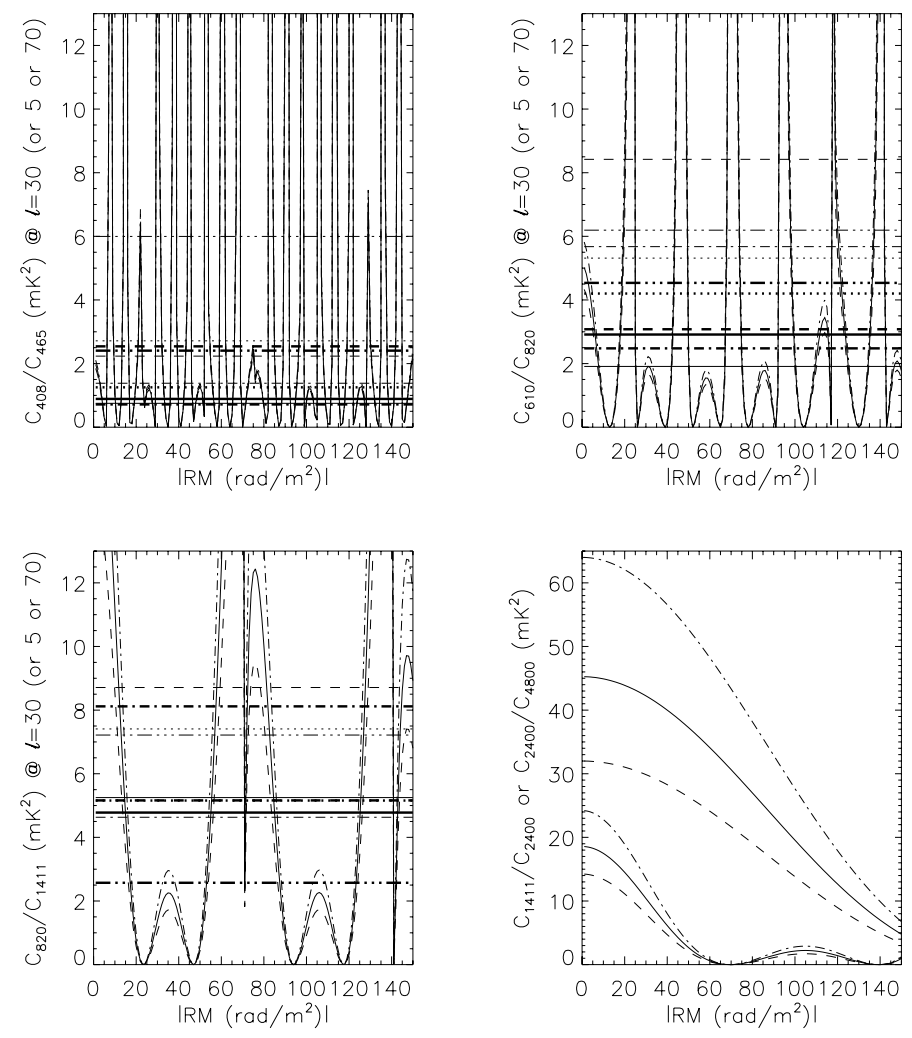

Fig. 10. Ratios $\left(C_{\tilde{\varphi}}^{\text {synch }}\left(v_{1}\right) / C_{\tilde{\ell}}^{\text {synch }}\left(v_{2}\right)\right)$ between the polarized synchrotron APSs at two frequencies for a pair of representative multipoles in the range of validity of our analysis. For the patches we plot the ratio at $\ell=30$ and 70 , whereas for the full coverage maps we chose $\ell=5$ and 30 . The horizontal lines represent the observed ratio corrected for beamwidth depolarization; thick lines refer to the lower of the two considered multipoles. Different sky regions are considered: full survey coverages (solid line), patch 1 (dots), patch 2 (dashes), patch 3 (dotdashes), patch 4 (three dots-dashes). The oscillating curves represent the theoretical ratio as a function of $R M$ in case of pure Faraday depolarization for three choices of the synchrotron emission frequency spectral index: $-(\beta+2)=-2.5$ (dashes), solid $-(\beta+2)=-2.75$ (solid lines), and $-(\beta+2)=-3$ (dot-dashes). The last panel show the same three curves in the hypothetical case of availability of observations at higher frequencies.

Exploiting the total intensity northern sky map at $1.4 \mathrm{GHz}$ (Reich \& Reich 1988), we estimate the mean polarization degree $\bar{\Pi}$ in the portion of the sky covered by the Leiden survey at $1411 \mathrm{MHz}$. We find $\bar{\Pi}^{\text {obs }} \sim 17,27,28,25,12 \%$ for the full coverage and for the patch 1, 2, 3, and 4, respectively. By using the preliminary DRAO survey, for the same sky regions we obtaine slightly lower values, i.e. $\bar{\Pi}^{\text {obs }} \sim 12,22,24,20$, and $9 \%$, respectively. Given the relation existing between the intrinsic and observed brightness polarized temperature in the slab model approximation, the observed degree of polarization can be written as:

$\Pi^{\mathrm{obs}}=\Pi^{\mathrm{intr}} \cdot|\sin \Delta \phi / \Delta \phi|$.

In Fig. 11 the maximum possible value of $\Pi^{\text {obs }}$ (derived assuming $\Pi^{\text {intr }}=0.75$ ) is displayed as a function of the $R M$. Each horizontal line represents the mean polarization degree actually 


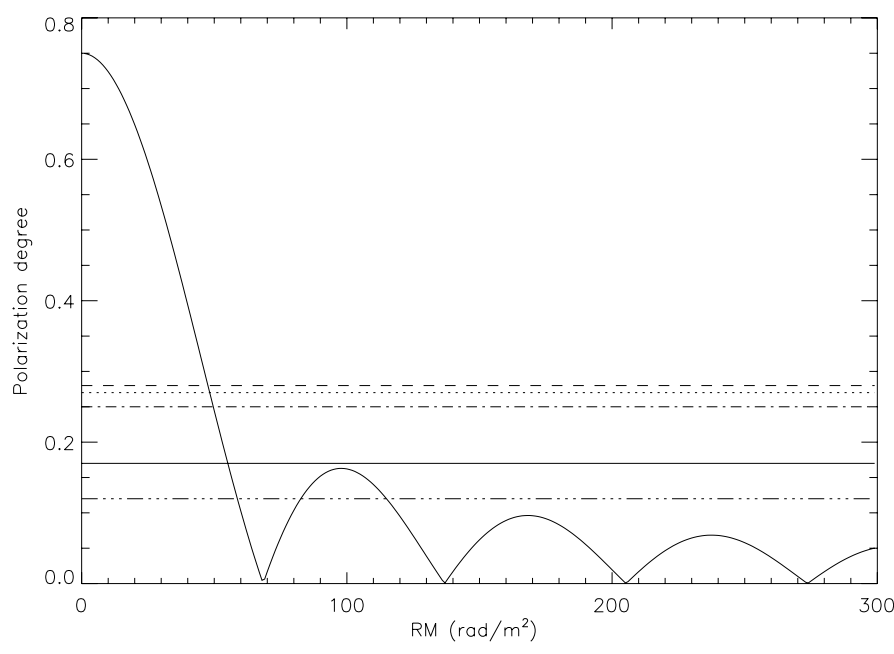

Fig. 11. The maximum possible value of the observed polarization degree (i.e. $\Pi^{\text {obs }}=\Pi^{\text {intr }} \cdot|\sin \Delta \phi / \Delta \phi|$, where $\Pi^{\text {intr }}=0.75$ ) is displayed as function of the $R M$. The horizontal lines represent the mean polarization degree observed in the considered sky regions. Survey full coverage (solid line), patch 1 (dots), patch 2 (dashes), patch 3 (dot-dash), patch 4 (three dots-dash).

observed for the Leiden surveys in the considered coverage cases $^{15}$.

Except for patch 4, the observed polarization degree sets an upper limit to the $R M$ of $\sim 50 \mathrm{rad} / \mathrm{m}^{2}$ (having assumed in Fig. 11 the maximum degree of polarization). Consequently the lowest of the $R M$ intervals previously identified (exploiting the APSs amplitude ratio at the two highest frequencies of the Leiden surveys) seems to be the most reasonable.

The above considerations hold under the following assumptions: $i)$ the physical properties of the magneto-ionic ISM responsible for depolarization are homogeneous along the line of sight; $i$ ) the bulk of the observed polarization signal comes spatially from the same regions at all frequencies. Under significant violations of these assumptions the meaning of derived $R M$ is by definition unclear.

The real situation is generally much more complicated, due to the existence of turbulence in both the Galactic magnetic field and the ISM electron density depolarizing the diffuse synchrotron emission (see Haverkorn et al. 2004). Another aspect is the presence of foreground magneto-ionic structures (Faraday screens) modulating the background synchrotron diffuse emission (see Wolleben \& Reich 2004; and Uyaniker 2003), which renders the understanding of the local ISM very difficult. There is a lack of information about the nature and spatial distribution of these structures and, consequently, about the corresponding depolarization effects.

Nevertheless, in patch 1, 2 and 3 the polarization vectors are extremely well aligned and similarly distributed at the two higher frequencies of the Leiden surveys. This observational fact suggests that for these areas the slab model is a reasonable approximation of the local ISM, at least for the angular scales

\footnotetext{
15 Note that the observed mean polarization degree might be due to the combination of Faraday depolarization and beam depolarization. If we were able to correct for beam depolarization effects, these lines would be shifted upwards in Fig. 11, leading to smaller values of RMs. Therefore the results of our analysis are rather conservative and provide upper limits to the RMs compatible with observations in the considered patches.
}

investigated in the present analysis. Consequently, the above considerations on the $R M \mathrm{~s}$ derived from the the APS ratios should be basically correct with respect to these regions (whereas they should be taken with caution in the other considered cases).

Finally, the right-bottom panel of Fig. 10 shows, as an example, that a multifrequency sky mapping at $v \sim$ some $\mathrm{GHz}$ would provide direct information on Faraday depolarization, significantly reducing the degeneracy on $R M$ (unavoidable in maps limited to $v \sim 1 \mathrm{GHz}$ ).

\subsection{Effect of the Faraday depolarization on the APS slope}

Our analysis of the Leiden surveys showed that the synchrotron emission APS steepens with increasing frequency (see Sect. 5). One possible explanation for such a behaviour of the APS relies on depolarization arguments: fluctuations of the electron density and/or of the magnetic field (in strength and/or direction) might redistribute the synchrotron emission power from the large to the small angular scales, creating fake structures. As a consequence the corresponding APS will become flatter, the effect being more important at the lower frequencies. Sticking to the slab model to describe the ISM, we simulated the Faraday depolarization effects on the APS at $408 \mathrm{MHz}$ and $1420 \mathrm{MHz}$ as follows. We assumed that the $1420 \mathrm{MHz}$ polarization map (Wolleben et al. 2006) represents the intrinsic Galactic synchrotron emission and used a spectral index of -2.75 to scale it down to $408 \mathrm{MHz}^{16}$. The polarization angle map at the two frequencies should be the same in the absence of depolarization effects; we adopted the polarization angle map at $1420 \mathrm{MHz}$ as intrinsic. These four maps are the input skies for our toy-simulation of Faraday depolarization. To build an $R M$ map we exploited the catalog produced by Spoelstra $(1984)^{17}$; it contains $\sim 1000 R M$ data, whose minimum and maximum angular distance is $d_{\min } \sim 0.02^{\circ}$ and $d_{\max } \sim 9^{\circ}$. We interpolated these data to generate an $R M$ map with $\theta_{\text {pixel }} \simeq 1.84^{\circ 18}$ (see Fig. 12); the algorithm searches in circles of increasing radius from each pixel centre, considering radii from $d_{\min }$ to $d_{\max }$. It stops as soon as it finds at least 3 points and associates to the pixel the weighted average of the corresponding values; the weight is inversely proportional to $d^{\mathrm{n}}$, where $d$ is the distance from the pixel center. The results we show below have been obtained choosing $n=1$, although we checked that they remain valid also for $n=0.5,2,4$.

\footnotetext{
16 The spectral index we used is rather steep for the polarized emission. However the present exercise is meant to justify the change of the APS slope with increasing frequency. It is not intended to reproduce the $408 \mathrm{MHz}$ map reconstructed from the Leiden data. This would require at least a frequency spectral index map appropriate to the polarization signal. The current knowledge of the spectral index distribution is limited to the total intensity radio diffuse Galactic emission and cannot be applied to the polarized emission, since the two come from different spatial regions.

17 The RM map obtained interpolating the values of Spoelstra (1984) are most likely not reliable. Those values were obtained performing a linear fit to the five frequencies data of the Leiden surveys and assuming as a result of the fit the lower possible RM. Furthermore beamwidth effects were considered to be negligible. Consequently the RMs derived by Spoelstra (1984) should be taken as indicative values. We simply exploited the existence of these data to perform toy-simulation of differential Faraday depolarization effects on the APSs of polarized emission.

18 The number of data points is about half the lowest one of the Leiden surveys catalogs, therefore we could not work with $\theta_{\text {pixel }} \simeq 0.92^{\circ}$.
} 

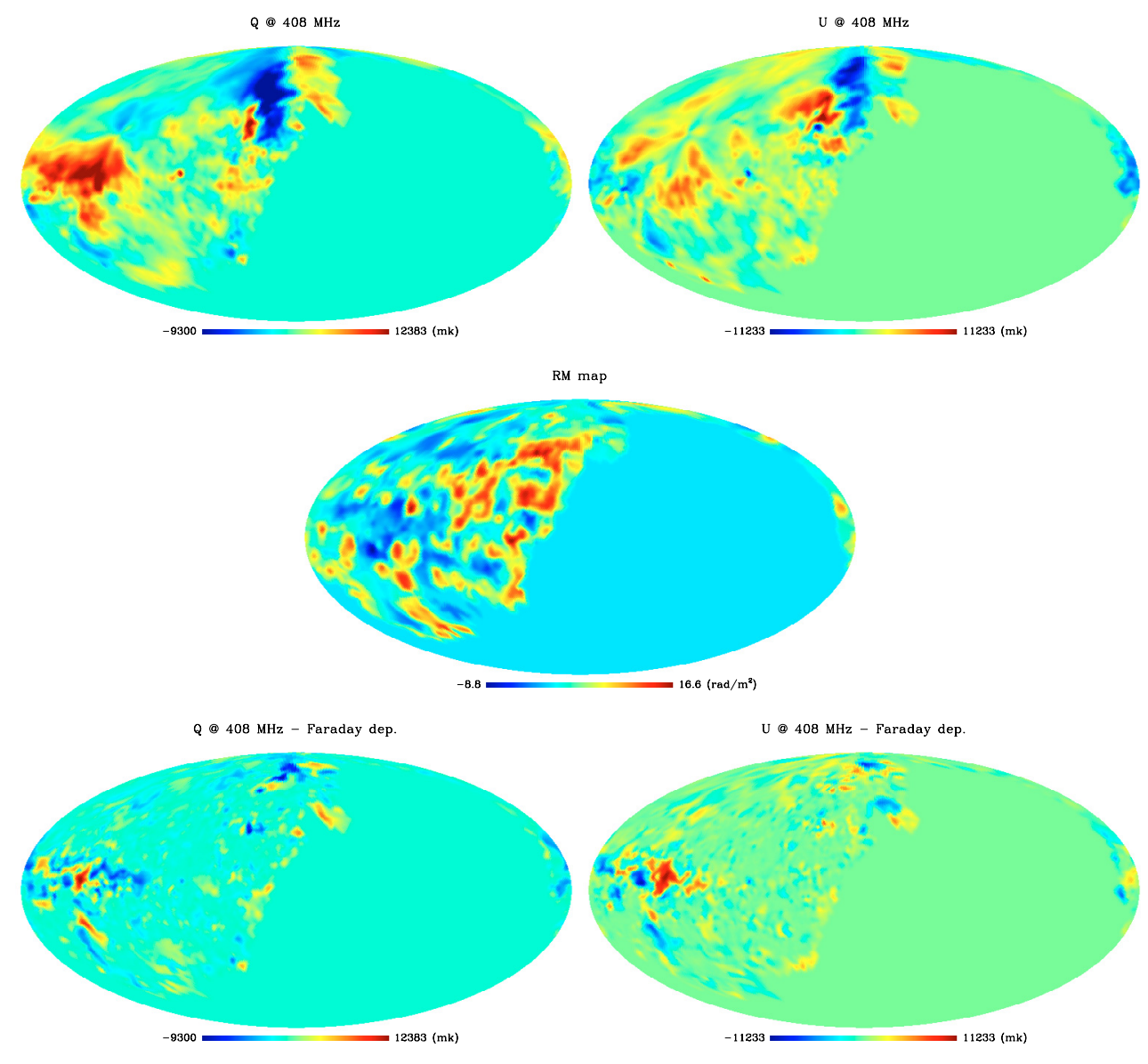

Fig. 12. Maps of the Stokes parameters before (upper panels) and after (lower panels) the transformation for Faraday depolarization. In the middle is placed the $R M$ map used in the simulation. All maps have been smoothed to $2.3^{\circ}$. In the depolarized maps the large scale structure appear significantly depressed respect to the input ones, whereas the small scale structures are more relevant.
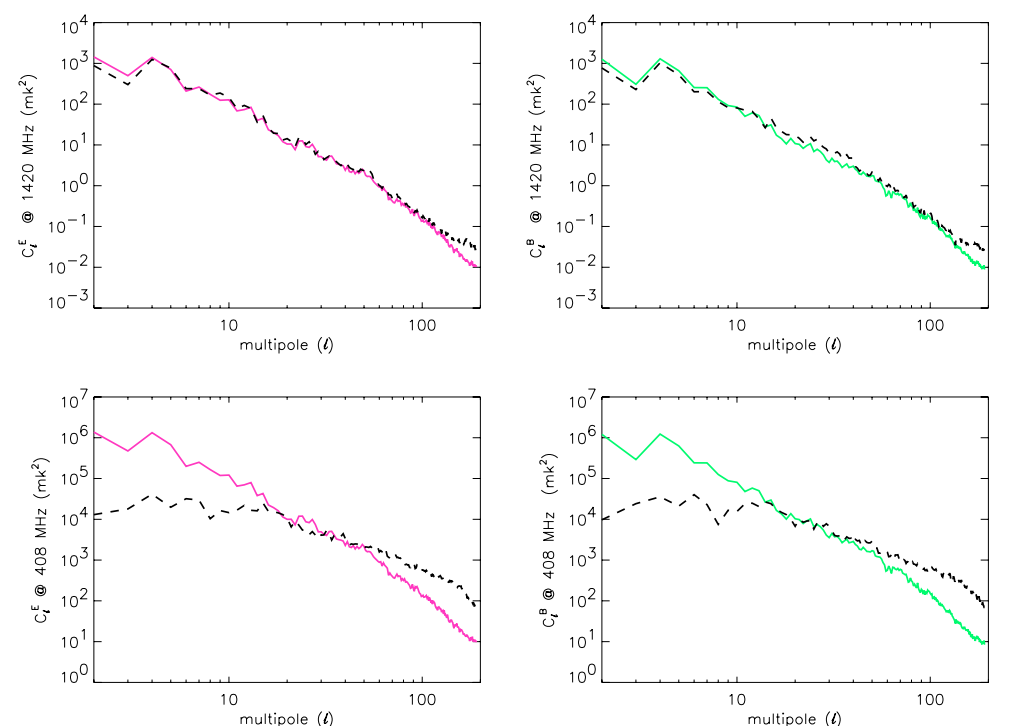

Fig. 13. $E$ and $B$ modes APSs before (solid lines) and after (dashed lines) the transformation according to Faraday depolarization. The upper panels refer to $1420 \mathrm{MHz}$ and the lower ones to $408 \mathrm{MHz}$. The different importance of the depolarization effects at the two frequencies is evident: the $1420 \mathrm{MHz}$ APSs are almost unaffected, whereas the $408 \mathrm{MHz}$ APSs significantly flatten.

We use the $R M$ map to transform the intrinsic $\phi$ and $P I$ for Faraday rotation and differential Faraday depolarization:

$\phi_{\text {output }} \rightarrow \phi_{\text {input }}+\Delta \phi$

$P I_{\text {output }} \rightarrow P I_{\text {input }} \cdot|\sin (\Delta \phi) / \Delta \phi|$.

At $1420 \mathrm{MHz}$ the input and output maps are very similar.
At $408 \mathrm{MHz}$ the situation is completely different: the large-scale structure appears significantly depressed in the depolarized map, whereas the small scale features become much more relevant (see Fig. 12).

In Fig. 13 we compare the APSs of the input and the output maps at both the frequencies. At $1420 \mathrm{MHz}$ the APSs are 
almost identical; on the contrary at $408 \mathrm{MHz}$ the depolarized map APSs significantly flatten, as expected from the increase of small scale features. This supports the interpretation of the bulk of the APS flattening with decreasing frequency resulting from our analysis of the Leiden surveys in terms of the Faraday depolarization effect.

\section{Discussion and conclusions}

The Leiden surveys have been up to now the only available data at $v \sim 1 \mathrm{GHz}$ suitable for a multifrequency study of the polarized diffuse component of the Galactic synchrotron emission on large angular scales. A linear polarization survey of the Southern sky had previously been carried out at $408 \mathrm{MHz}$ using the Parkes telescope (Mathewson \& Milne 1965); unfortunately these data have never been put in digital form. In Fig. 14 an image of the data from this survey has been combined with the Leiden survey at $408 \mathrm{MHz}$ to show an all-sky linear polarization map.

The "North Polar Spur", the "Fan Region", and the "Cetus Arc" (see Berkhuijsen 1971 for a sketch of the Galactic Loops these structures are thought to belong to) are visible as areas where the polarization vectors are longer (the length is proportional to the polarized intensity) and more regularly aligned, drawing small circles in the sky. The most plausible theory suggests that these Galactic spurs are the brighter ridges of evolved Supernova Remnant sitting relatively close to the Sun (within a few $\times 10^{2} \mathrm{pc}$ ) and obscuring our view of the Galactic diffuse non-thermal emission. The APSs obtained for these regions will be characterized by an amplitude considerably higher than the average, representing therefore an upper limit for the polarized signal of the Galactic synchrotron radiation. On the other hand, the APS should be steeper in these regions, since the polarization vectors appear rather ordered and with approximately constant length, implying a relatively low fluctuation power at small scales. These considerations should be kept in mind when extrapolating the results to the microwave range in order to check the impact of the Galactic synchrotron emission on CMB polarization measurements.

Implementing an interpolation method to deal with the sparse and irregular sampling of the Leiden surveys, we produced maps of $P I, Q$ and $U$ with $\theta_{\text {pixel }} \simeq 0.92^{\circ}$ at five frequencies between 408 and $1411 \mathrm{MHz}$. We accomplished several tests in order to check the reliability of the interpolation algorithm and to optimize its quality (see Sects. 3 and 4). We emphasize that the APSs computed from our maps are significantly different from those obtained by other authors (e.g. Bruscoli et al. 2002) for similar areas of the sky, using the same data; namely, at the higher frequencies of the Leiden surveys we find steeper APSs (first recognized in Burigana \& La Porta 2002). A firm confirmation of our approach comes from the comparison of the APSs obtained for the $1411 \mathrm{MHz}$ map with those of a preliminary version of the DRAO $1420 \mathrm{MHz}$ survey, characterized by much denser sampling and higher sensitivity.

We used the interpolated full coverage maps to study the APS of the polarized synchrotron emission on the multipole range $[2,50]$, being limited at high $\ell$ by the map sensitivity (related to the accuracy and sampling of the original data). Exploiting some better sampled regions, approximately coinciding with the two most prominent Galactic spurs, we could extend our analysis up to $\ell \sim 100$.

At each frequency the APSs of the entire coverage maps turn out to change slope at $\ell \sim 10, C_{\ell}^{P I}, C_{\ell}^{E}$, and $C_{\ell}^{B}$ presenting a steepening going from the lower to the higher multipoles (see Sect. 5). A general and remarkable result (see Sect. 5) is that

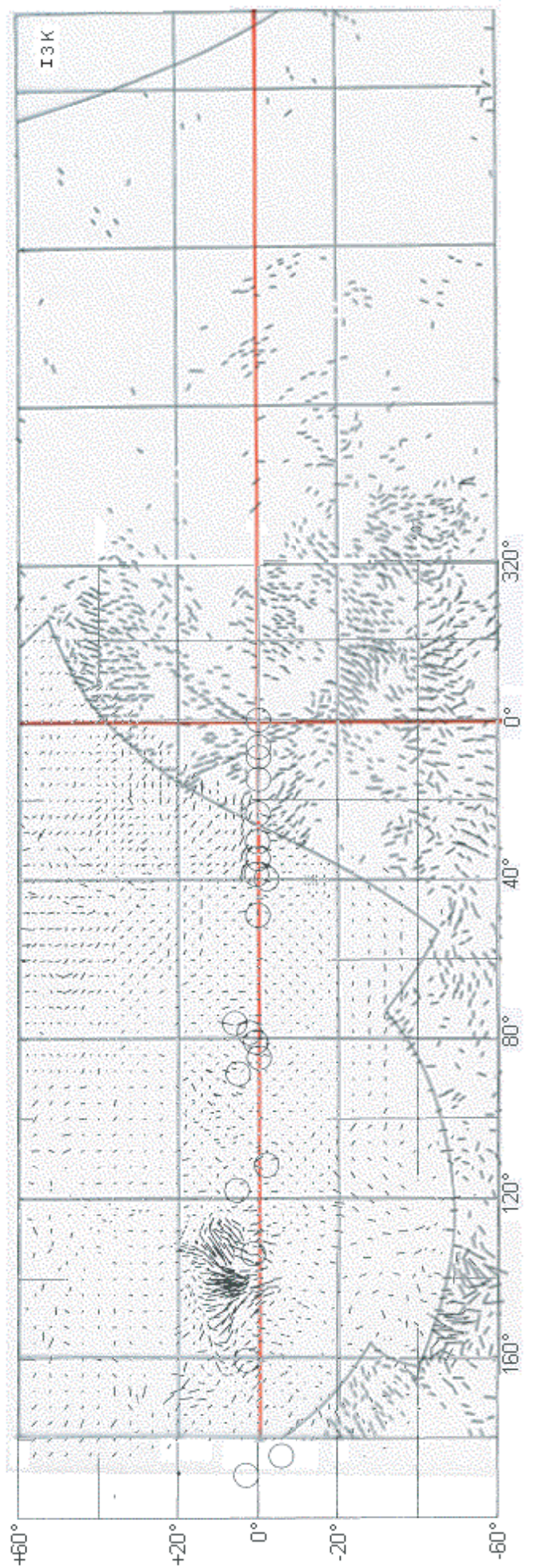

Fig. 14. Map of polarization vectors at $408 \mathrm{MHz}$, obtained combining the corresponding figures of the Leiden survey (Brouw \& Spoelstra 1976) and of the Parkes southern sky survey (Mathewson \& Milne 1965). Unfortunately, the vector length has a different scale in the two original figures, therefore only a direct comparison of the direction is feasible.

the slope of the polarized synchrotron emission APS increases from $408 \mathrm{MHz}$ to $1411 \mathrm{MHz}$; it changes from $\alpha \sim-(1-1.5)$ to $\alpha \sim-(2-3)$ for $\ell \sim 10$ to $\sim 100$ and from $\alpha \sim-0.7$ to 
$\alpha \sim-1.5$ for lower multipoles, the exact value depending on the considered sky region and polarization mode.

One possible explanation for such a behaviour of the APS relies on Faraday depolarization arguments. Fluctuations of the electron density and/or of the magnetic field (in strength and/or direction) might redistribute the synchrotron emission power from the larger to the smaller angular scales, creating fake structures. The effect is more relevant at lower frequencies, where the APS slope is expected to become flatter. We have verified this interpretation by performing a toy-simulation of pure Faraday depolarization in a simple slab model. We note that in the better sampled patches the polarization vectors are well ordered and similarly distributed at $820 \mathrm{MHz}$ and $1411 \mathrm{MHz}$, which is consistent with the slab model assumptions at least for the considered angular scales. We reproduced the effects of Faraday depolarization on simulated polarized intensity maps, representing the intrinsic Galactic synchrotron emission at $408 \mathrm{MHz}$ and $1420 \mathrm{MHz}$. We constructed an $R M$ map interpolating the data of Spoelstra (1984) and applied it to transform the input maps according to Faraday depolarization formulae. At $1420 \mathrm{MHz}$ the input and output maps are almost identical, whereas at $408 \mathrm{MHz}$ the large-scale structure of the output map appears depressed and the small scale structures more abundant. Consequently, the corresponding $408 \mathrm{MHz}$ APSs are flatter than the original ones (see Sect. 7.2 for a more precise description of the simulation process). It is therefore preferable to extrapolate the results (spectral index and amplitude of the APS) obtained at $1411 \mathrm{MHz}$ (significantly less affected by Faraday depolarization effect than those at $408 \mathrm{MHz}$ ) to estimate the synchrotron APS at microwave frequencies.

By interpreting the ratios of the APS amplitudes at 820 and $1411 \mathrm{MHz}$ (the latter smoothed to the lower angular resolution of the former) in terms of synchrotron emission depolarized by Faraday effect, we have identified possible $R M$ ranges (see Sect. 7.1). This seems a reasonable approach at least in the case of patch 1,2, and 3, since the polarization vectors are mostly aligned, indicating rather homogeneous physical conditions. Furthermore, taking into account the upper limit to $R M$ derived from the available information on the degree of polarization at $1.4 \mathrm{GHz}$ and considering that the maximum theoretical degree of synchrotron polarization is $\sim 75 \%$, we could break or, at least, reduce the degeneracy between the identified $R M$ intervals. For the three better sampled patches, the most reasonable value of $R M$ are $\sim 9-17 \mathrm{rad} / \mathrm{m}^{2}$. However, given the uncertainty on the measured polarization degree, $R M$ values in the interval $\sim 53-59 \mathrm{rad} / \mathrm{m}^{2}$ cannot be excluded. Higher frequencies observations (at $v \gtrsim 2-3 \mathrm{GHz}$ ) would be extremely useful to break the degeneracy left by the available $\sim 1 \mathrm{GHz}$ data.

Acknowledgements. We are grateful to W. Reich for many constructive discussions on the topic and for a careful reading of the original manuscript. We aknowledge M. Tucci and P. Leahy and thank T. A. T. Spoelstra, R. Wielebinski and R. Beck. L.L.P. thanks E. Hivon. C.B. thanks W. Reich, R. Wielebinski, and the MPIfR, Bonn for the warm hospitality. L.L.P. was supported for this research through a stipend from the International Max Planck Research School (IMPRS) for Radio and Infrared Astronomy at the Universities of Bonn and Cologne. Some of the results in this paper have been derived using the HEALPix package (Górski et al. 2005). We warmly thank the anonymous referee for useful comments.

\section{References}

Baccigalupi, C., Bedini, L., Burigana, C., et al. 2000, MNRAS, 318, 769 Baccigalupi, C., Burigana, C., Perrotta, F., et al. 2001, A\&A, 372, 8 Baccigalupi, C., Perrotta, F., De Zotti, G., et al. 2004, MNRAS, 354, 55 Barnes, C., Hill, R. S., Hinshaw, G., et al. 2003, ApJS, 148, 51 Berkhuijsen, E. 1971, A\&A, 14, 252

Bingham, R. G. 1967, MNRAS, 137, 157

Bouchet, F. R., Prunet, S., \& Sethi, S. 1999, MNRAS, 302, 663

Brouw, W. N., \& Spoelstra, T. A. T. 1976, A\&AS, 26, 129

Burigana, C., \& La Porta, L. 2002, in Astrophysical Polarized Background, ed. S. Cecchini, S. Cortiglioni, R. Sault, \& C. Sbarra, AIP Conf. Proc., 609, 54 [arXiv: astro-ph/0202439]

Burigana, C., Maino, D., Gòrski, K. M., et al. 2001, A\&A, 373, 345

Bruscoli, M., Tucci, M., Natale, V., et al. 2002, NewA, 7, 171

Burigana, C., \& Sáez, D. 2003, A\&A, 409, 423

Burigana, C., Finelli, F., Salvaterra, R., Popa, L. A., \& Mandolesi, N. 2004,

Recent Research Developments in Astronomy \& Astrophysics, 2, 59

Burn, B. J. 1966, MNRAS, 133, 67

Challinor, A., Fosalba, P., Mortlock, D., et al. 2000, Phys. Rev. D, 62, 15

Delabrouille, J., Cardoso, J.-F., \& Patanchon, G. 2003, MNRAS, 346, 1089

Egger, R. J., \& Aschenbach, B. 1995, A\&A, 294, L25

Gardner, F. F., \& Whiteoak, J. B. 1966, ARA\&A, 4, 245

Giardino, G., Banday, A. J., Górski, K. M., et al. 2002, A\&A, 387, 82

Ginzburg, V. L., \& Syrovatski, S. I. 1965, ARA\&A, 3, 297

Górski, K. M., Hivon, E., Banday, A. J., et al. 2005, ApJ, 622, 759

Haverkorn, M., Katgert, P., \& de Bruyn, A. G. 2004, A\&A, 427, 549

Hobson, M. P., Jones, A. W., Lasenby, A. N., et al. 1998, MNRAS, 300, 1

Kamionkowski, M., Kosowsky, A., \& Stebbins, A. 1997, Phys. Rev. D, 55, 7368

Kosowsky, A. 1999, NewAR, 43, 157

Kovac, J. 2002, Nature, 420, 772

La Porta, L. 2001, Graduation Thesis, Astronomy Department, Bologna University, Italy

La Porta, L., Burigana, C., Reich, W., \& Reich, P. 2006, A\&A Lett., in press

Maino, D., Farusi, A., Baccigalupi, C., et al. 2002, MNRAS, 334, 53

Masi, S., Ade, P. A. R., Bock, J. J., et al. 2006, A\&A, accepted [arXiv:astro-ph/0507509]

Mathewson, D. S., \& Milne, D. K. 1965, Aust. J. Phys, 18, 635

Montroy, T. E., Ade, P. A. R., Bock, J. J., et al. 2005, ApJ, submitted [arXiv:astro-ph/0507514]

Peebles, P. J. E. 1993, Principles of Physical Cosmology (Princeton University Press)

Platania, P., Bensadoun, M., Bersanelli, M., et al. 1998, ApJ, 505(2), 473

Reich, P., \& Reich, W. 1988, A\&AS, 74, 7

Salter, C. J. 1983, BASI, 11, 1

Smoot, G. F., Bennett, G., Weber, R., et al. 1990, ApJ, 360, 685

Sokoloff, D. D., Bykov, A. A., Shukurov, A., et al. 1998, MNRAS, 299, 1985

Spoelstra, T. A. T. 1984, A\&AS, 135, 238

Tauber, J. A. 2004, AdSpR, 34, 91

Testori, J. C., Reich, P., Bava, J. A., et al. 2001, A\&A, 368, 1123

Testori, J. C., Reich, P., \& Reich, W. 2003, in The Magnetized Interstellar Medium, ed. B. Uyaniker, W. Reich, \& R. Wielebinski (KatlenburgLindau: Copernicus GmbH), 57, http://www.mpifr-bonn.mpg.de/div/ konti/antalya/contrib.html

Uyaniker, B., 2003, in The Magnetized Interstellar Medium, ed. B. Uyaniker, W. Reich, \& R. Wielebinski (Katlenburg-Lindau: Copernicus GmbH), 71, http: //www .mpifr-bonn.mpg.de/div/konti/antalya/contrib.html Uyaniker, B., Furst, E., Reich, W., et al. 1998, A\&A, 132, 401

Vinyajkin, E. N., \& Razin, V. A. 2002, in Astrophysical Polarized Background, AIP Conf. Proc., 609, 26

Wolleben, M. 2005, Ph.D. Thesis, Bonn University, Germany

Wolleben, M., \& Reich, W. 2004, A\&A, 427, 537

Wolleben, M., Landecker, T. L., Reich, W., \& Wielebinski, R. 2003, in The Magnetized Interstellar Medium, ed. B. Uyaniker, W. Reich, \& R. Wielebinski (Katlenberg-Lindau: Copernicus $\mathrm{GmbH}$ ), 51,

http: //www .mpifr-bonn.mpg.de/div/konti/antalya/contrib.html Wolleben, M., Landecker, T. L., Reich, W., \& Wielebinski, R. 2006, A\&A, 448, 411

Zaldarriaga, M. 2001, Phys. Rev. D, 64, 15 